\title{
Appraising the Early-est earthquake monitoring system for tsunami alerting at the Italian Candidate Tsunami Service Provider
}

\author{
F. Bernardi ${ }^{1}$, A. Lomax ${ }^{2}$, A. Michelini ${ }^{1}$, V. Lauciani ${ }^{1}$, A. Piatanesi ${ }^{1}$, and S. Lorito ${ }^{1}$ \\ ${ }^{1}$ Istituto Nazionale di Geofisica e Vulcanologia, Via di Vigna Murata 605, 00143 Rome, Italy \\ ${ }^{2}$ ALomax Scientific, Allé du Micocoulier 161, 06370 Mouans-Sartoux, France \\ Correspondence to: F. Bernardi (fabrizio.bernardi@ingv.it)
}

Received: 16 March 2015 - Published in Nat. Hazards Earth Syst. Sci. Discuss.: 30 April 2015

Revised: 12 August 2015 - Accepted: 13 August 2015 - Published: 11 September 2015

\begin{abstract}
In this paper we present and discuss the performance of the procedure for earthquake location and characterization implemented in the Italian Candidate Tsunami Service Provider at the Istituto Nazionale di Geofisica e Vulcanologia (INGV) in Rome. Following the ICG/NEAMTWS guidelines, the first tsunami warning messages are based only on seismic information, i.e., epicenter location, hypocenter depth, and magnitude, which are automatically computed by the software Early-est. Early-est is a package for rapid location and seismic/tsunamigenic characterization of earthquakes. The Early-est software package operates using offline-event or continuous-real-time seismic waveform data to perform trace processing and picking, and, at a regular report interval, phase association, event detection, hypocenter location, and event characterization. Early-est also provides $\mathrm{mb}, M_{\mathrm{wp}}$, and $M_{\mathrm{wpd}}$ magnitude estimations. mb magnitudes are preferred for events with $M_{\mathrm{wp}} \lesssim 5.8$, while $M_{\mathrm{wpd}}$ estimations are valid for events with $M_{\mathrm{wp}} \gtrsim 7.2$. In this paper we present the earthquake parameters computed by Early-est between the beginning of March 2012 and the end of December 2014 on a global scale for events with magnitude $M \geq 5.5$, and we also present the detection timeline. We compare the earthquake parameters automatically computed by Early-est with the same parameters listed in reference catalogs. Such reference catalogs are manually revised/verified by scientists. The goal of this work is to test the accuracy and reliability of the fully automatic locations provided by Early-est. In our analysis, the epicenter location, hypocenter depth and magnitude parameters do not differ significantly from the values in the reference catalogs. Both $\mathrm{mb}$ and $M_{\mathrm{wp}}$ magnitudes show differences to the reference catalogs. We thus derived correction functions in order to minimize the differences and
\end{abstract}

correct biases between our values and the ones from the reference catalogs. Correction of the $M_{\mathrm{wp}}$ distance dependency is particularly relevant, since this magnitude refers to the larger and probably tsunamigenic earthquakes. $M_{\mathrm{wp}}$ values at stations with epicentral distance $\Delta \lesssim 30^{\circ}$ are significantly overestimated with respect to the CMT-global solutions, whereas $M_{\text {wp }}$ values at stations with epicentral distance $\Delta \gtrsim 90^{\circ}$ are slightly underestimated. After applying such distance correction the $M_{\mathrm{wp}}$ provided by Early-est differs from CMT-global catalog values of about $\delta M_{\mathrm{wp}} \approx 0.0 \mp 0.2$. Early-est continuously acquires time-series data and updates the earthquake source parameters. Our analysis shows that the epicenter coordinates and the magnitude values converge within less than $10 \mathrm{~min}$ ( $5 \mathrm{~min}$ in the Mediterranean region) toward the stable values. Our analysis shows that we can compute $M_{\mathrm{wp}}$ magnitudes that do not display short epicentral distance dependency overestimation, and we can provide robust and reliable earthquake source parameters to compile tsunami warning messages within less than $15 \mathrm{~min}$ after the event origin time.

\section{Introduction}

Tsunamis may produce dangerous coastal flooding and inundations accompanied by powerful currents which can cause significant damage and casualties. A tsunami may be generated when a large or great earthquake occurs in oceans or inland close to the coast. When such earthquakes occur, a tsunami warning should be issued to alert national authorities and emergency management officials to take action for the entire tsunami hazard zone, such as evacuating the pop- 
ulation or securing critical facilities such as nuclear power plants. With advance evacuation plans and well-informed communities, tsunami warnings could also be sent directly to the population.

Reliable tsunami warnings should be disseminated as fast as possible in order to also be effective for the coastal areas very close to the earthquake source, since a tsunami may arrive at these areas within the first few minutes after the event origin time. Populations exposed to tsunami hazards in the field near to the source, however, should be aware that the time between warning issuance and tsunami impact may be too short to escape the tsunami; warning may arrive even after the tsunami, or the system may be subject to failure for several reasons. Hence, the population should know how to self-evacuate relying on natural warnings when they are present, such as strong and/or unusually long shaking, ocean withdrawal, an anomalously rising tide, roaring sounds from the ocean, etc.

To provide the earliest possible alerts, initial warnings from regional tsunami warning systems are normally only based on seismic information. Thus, fast, precise, and reliable earthquake source parameters like epicenter coordinates, hypocenter depth, and magnitude are crucial for seismologically based tsunami early warning procedures. This is particularly important in the Mediterranean Sea, where the tsunami wave travel times between source regions and coastlines are short and dedicated deep-sea instruments, such as DART ${ }^{\circledR}$ buoys (http://nctr.pmel.noaa.gov/Dart/), to be in place.

The Istituto Nazionale di Geofisica e Vulcanologia (INGV) in Italy is a Candidate Tsunami Service Provider (CTSP) in the framework of ICG/NEAMTWS (NEAMTWS, 2011), which is the tsunami early warning and mitigation system established by IOC/UNESCO for the northeastern Atlantic, the Mediterranean and connected seas. For this reason, the Centro Allerta Tsunami (CAT) (Italian for "tsunami alert center"), was established at the INGV headquarter in Rome at the end of 2013. The CAT mission is to implement and maintain a $24 / 7$ service alongside the ordinary seismic surveillance of the national territory, and to work towards a probabilistic seismic hazard assessment (PSHA) for the Italian coasts, that is a tsunami hazard map for seismically induced tsunamis (Basili et al., 2013). CAT-INGV started operations on a 24/7 basis as a CTSP in October 2014. Monthly communication tests are performed with national authorities, subscriber IOC member states, and other institutions, such as the DG-ECHO Emergency Response Coordination Center in Brussels. In the NEAM region there are three other CTSPs in operation: CENALT in France, NOA in Greece, and KOERI in Turkey. IPMA, in Portugal, should begin operations soon. Each of these CTSPs has its specific competence source areas within the NEAM region.

At the national level, INGV is responsible for issuing messages to the Civil Protection authority, which is presently responsible for alert dissemination. INGV also maintains the national seismic network and exchanges seismic data in real time with a number of international seismic data providers. The Istituto Superiore per la Protezione e Ricerca Ambientale (ISPRA) maintains the national sea level network and provides real-time data to the INGV monitoring room. The implemented tsunami warning procedure uses the Earlyest software developed by Lomax and Michelini (2009a, b, $2011,2012)$ to rapidly detect, locate, and determine the magnitude for large to great regional and teleseismic earthquakes.

The purpose of this paper is to analyze the performance of Early-est regarding past events, in order to evaluate its reliability for the near-real-time tsunami warnings disseminated by the INGV, and eventually tune the procedure as a whole.

INGV CTSP follows the ICG/NEAMTWS guidelines. ICG/NEAMTWS rules establish that a CTSP must disseminate a tsunami message, with warning levels that depend on location, magnitude, and depth of the earthquake according to a decision matrix, for all earthquakes with magnitudes $M \geq 5.5$ in their zone of competence. Messages are sent for earthquakes that are large and shallow enough, and which occur in sea areas or inland but are sufficiently close to the coast to possibly generate a tsunami. INGV is responsible for the earthquake and tsunami source zone extending from the Gibraltar Strait in the west, to Marmara and Levantine seas to the east.

The seismicity in the Mediterranean region is moderate to high but also includes $M=8+$ earthquakes that occurred in the past and generated significant tsunamis (Maramai et al., 2014; Lorito et al., 2015). It is difficult to assess if $M=9$ class earthquakes might occur, and these can not be excluded (Kagan and Jackson, 2013). Even if tsunamigenic earthquakes are likely to occur, their time recurrence intervals are however quite long (Koravos et al., 2003; Jenny et al., 2004; Bungum and Lindholm, 2007); moreover, the Mediterranean Sea is a relatively small area, and earthquakes with $M \geq 5.5$ do not occur very frequently. The Global CMT catalogs (Dziewonski et al., 1981; Ekström et al., 2012) include about 125 earthquakes with $M_{\mathrm{w}} \geq 5.5$ within the Mediterranean region, which implies an occurrence rate of $\approx 30 \mathrm{ev}-$ ery 10 years. Early-est has now been running for several years, but only since the beginning of March 2012 has its current major version release been online and its solutions have been able to be systematically archived; thus we have few events to analyze for tuning our tsunami alert procedure (Table 1). For this reason, we perform our analysis using all earthquakes which have occurred worldwide and have been located by Early-est since March 2012. To perform the analysis and tune our procedure, we proceed by comparing the epicenters, the hypocenter depths, and the estimation of magnitudes provided fully automatically by Early-est with the same parameters provided by other agencies taken as a reference. Such agencies provide manually validated/revised locations and magnitude estimations for earthquakes on a global scale.

This paper is structured as follows: in the next section, we give a brief overview of the Early-est algorithm, in Sect. 3 we 
Table 1. List of earthquakes that occurred in the Mediterranean region located by Early-est with $M \geq 5.5$ between March 2012 and December 2014. For each event we have listed the computed event origin time, epicenter coordinates, hypocenter depth, the maximum $68 \%$ confidence error in $x y z$ space (in kilometers), the preferred magnitude ( $\mathrm{mb}, M_{\mathrm{wp}}$ or $M_{\mathrm{wpd}}$ ), and a reference magnitude, i.e., when the first Early-est locations were available (in seconds) after the event origin time, and when the magnitudes stabilize (in minutes) after the first location was available. A magnitude is stable when the difference to the final magnitude is $\leq \mp 0.2$.

\begin{tabular}{rcccccccccc}
\hline No. & Date & Time & Lat. & Long. & Depth & $\delta(x y z)$ & Mag $^{\text {best }}$ & Mag $^{\text {ref }}$ & $\begin{array}{c}\text { First } \\
\text { location }\end{array}$ & $\begin{array}{c}\text { First } \\
\text { magnitude }\end{array}$ \\
\hline 1 & $2012-06-10$ & $12: 44: 15$ & 36.36 & 28.93 & 19.7 & 4.3 & $M_{\mathrm{wp}}=6.1$ & $M_{\mathrm{W}}^{\mathrm{CMT}}=6.1$ & 167 & 10 \\
2 & $2012-09-12$ & $03: 27: 43$ & 34.77 & 24.08 & 10.0 & 5.1 & $\mathrm{mb}=5.7$ & $\mathrm{mb}^{\mathrm{Nc}}=5.4$ & 201 & 7 \\
3 & $2013-01-08$ & $14: 16: 09$ & 39.62 & 25.49 & 10.1 & 4.2 & $M_{\mathrm{wp}}=5.7$ & $M_{\mathrm{W}}^{\mathrm{CMT}}=5.7$ & 174 & 3 \\
4 & $2013-06-15$ & $16: 11: 02$ & 34.51 & 24.99 & 15.4 & 5.4 & $M_{\mathrm{wp}}=6.4$ & $M_{\mathrm{W}}^{\mathrm{CMT}}=6.3$ & 181 & 2 \\
5 & $2013-06-16$ & $21: 39: 07$ & 34.51 & 25.00 & 18.6 & 4.8 & $M_{\mathrm{wp}}=6.1$ & $M_{\mathrm{W}}^{\mathrm{CMT}}=6.0$ & 117 & 3 \\
6 & $2013-10-12$ & $13: 11: 51$ & 35.52 & 23.30 & 11.5 & 5.2 & $M_{\mathrm{wp}}=6.6$ & $M_{\mathrm{W}}^{\mathrm{CMT}}=6.8$ & 194 & 2 \\
7 & $2013-12-28$ & $15: 21: 06$ & 36.04 & 31.30 & 56.8 & 8.5 & $M_{\mathrm{wp}}=6.0$ & $M_{\mathrm{W}}^{\mathrm{CMT}}=5.9$ & 358 & 5 \\
8 & $2014-01-26$ & $18: 45: 10$ & 38.29 & 20.38 & 19.8 & 2.5 & $\mathrm{mb}=5.2$ & $M_{\mathrm{W}}^{\mathrm{Nc}}=5.4$ & 115 & 3 \\
9 & $2014-02-03$ & $03: 08: 46$ & 38.25 & 20.40 & 10.1 & 2.3 & $M_{\mathrm{wp}}=6.1$ & $M_{\mathrm{W}}^{\mathrm{CMT}}=6.0$ & 77 & 7 \\
10 & $2014-04-04$ & $20: 08: 07$ & 37.26 & 23.71 & 115.9 & 2.2 & $\mathrm{mb}=5.5$ & $M_{\mathrm{W}}^{\mathrm{CMT}}=5.6$ & 119 & 6 \\
11 & $2014-05-24$ & $09: 25: 03$ & 40.23 & 25.34 & 10.1 & 4.8 & $M_{\mathrm{wp}}=6.6$ & $M_{\mathrm{W}}^{\mathrm{CMT}}=6.9$ & 124 & 7 \\
12 & $2014-08-29$ & $03: 45: 06$ & 36.75 & 23.67 & 81.2 & 2.7 & $M_{\mathrm{wp}}=5.8$ & $M_{\mathrm{W}}^{\mathrm{CMT}}=5.8$ & 119 & 4 \\
\hline
\end{tabular}

Table 2. Global earthquake catalogs used for the analysis in this work. For each catalog we have indicated the begin and end time of the time window of the data set included in this work. Catalog abbreviations used in this paper are in brackets in the first column.

\begin{tabular}{lllr}
\hline Catalog & Begin & End & Type \\
\hline Early-est (EEc) & $03-2012$ & $12-2014$ & automatic \\
NEIC (Nc) & $01-2004$ & $12-2014$ & revised \\
GFZ (Gc) & $06-2006$ & $12-2014$ & revised \\
CSEM (Cc) & $10-2004$ & $12-2014$ & revised \\
PTWC (Pc) & $12-2013$ & $06-2014$ & revised \\
CMT-Harvard (CMT) & $01-1976$ & $10-2014$ & revised \\
\hline
\end{tabular}

describe the data set used in our analysis, and in the three sections following that, we then analyze and compare the earthquake source parameters provided by Early-est with the ones provided by the reference agencies; first the epicenter location (Sect. 4), then the hypocenter depth (Sect. 5), and lastly the magnitude (Sect. 6). In Sect. 7 we will analyze the speed performances of Early-est with respect to the location and the magnitude parameters, in order to set the timeline of our automatic tsunami warning procedure. Lastly, we present the discussions and conclusions.

\section{Early-est algorithm description}

Early-est is a software package for rapid location and seismic/tsunamigenic characterization of earthquakes. The Early-est software package operates using offline-event or continuous-real-time seismic waveform data to perform trace processing and picking, and, at a regular report interval, phase association, event detection, hypocenter location, and event characterization. This characterization (Table A1) includes $\mathrm{mb}$ and $M_{\mathrm{wp}}$ magnitudes, the determination of apparent rupture duration, $T_{0}$, large earthquake magnitude, $M_{\mathrm{wpd}}$, and the assessment of tsunamigenic potential using $T_{d}$ and $T_{50} \mathrm{Ex}$, as described in Lomax and Michelini (2009a, b, 2011). The Early-est program reads Mini-SEED data packets from a file or a SeedLink server (http://ds.iris. edu/ds/nodes/dmc/services/seedlink, http://www.seiscomp3. org/wiki, doc/applications/seedlink), and passes each packet to a trace-processing module. The program also runs an associate/locate-reporting module at regular reporting intervals (e.g., after all data are read by Mini-SEED; every 1 min for SeedLink). The Early-est software maintains a persistent pick list for the current reporting window (e.g., the last hour before real time) and an event list for a specified archive interval (e.g., the last 10 days). The pick list is updated continuously as picking and trace processing are applied to new data packets. The event list is updated at each reporting interval as new event locations are found or previous locations are deleted. At each reporting interval, the associate/locate module processes the current pick list from scratch, without making use of previous associations or location information from the event list; this memory-less procedure simplifies the associate/locate module and makes it very robust with respect to changes in the pick list, but increases the computational load. To reduce this load, the persistence of association and location information for well located events is currently being added to Early-est. 


\subsection{Trace-processing module}

The trace-processing module processes each new data packet passed by the Early-est program. This processing includes channel identification, quality control, filtering for picking, picking, and further filtering and pre-processing as required for seismic and tsunamigenic event characterization (Table A1).

Picking in Early-est is performed by FilterPicker (Lomax and Michelini, 2012; Vassallo et al., 2012), a general purpose, broadband, phase detector and picker which is applicable to real-time seismic monitoring and earthquake early warning. FilterPicker uses an efficient algorithm which operates stably on continuous, real-time, broadband signals, avoids excessive picking during large events, and produces onset timing, realistic timing uncertainty, onset polarity, and amplitude information. In practice, it operates on a predefined number of frequency bands by generating a set of bandpassed time series with different center frequencies. Characteristic functions are determined for each frequency band and a pick is declared if and when, within a window of predefined time width, the integral of the maximum of the characteristic functions exceeds a predefined threshold.

After picking for each new data packet, for each pick in the pick list for the current packet channel, the trace-processing module applies various analyses on the channel data and updates values needed for event characterization. Recursive, time-domain algorithms are used for all filtering and other time-series processing.

\subsection{Associate/locate-reporting module}

The Early-est associate/locate-reporting module runs an octree associate/locate module with the current pick list, and then the reporting module which determines event characterization results and generates graphical and alpha-numeric reporting output. The octree associate/locate module efficiently and robustly associates picks, and detects and locates seismic events over the whole Earth from 0 to $700 \mathrm{~km}$ depth using the efficient, nonlinearized, probabilistic and global, octree importance-sampling search (Lomax et al., 2001, 2009). See Appendix A for more details.

The Early-est reporting module processes the current pick list and event list to determine event characterization results (Table A1) and generate graphical, alpha-numeric, XML, HTML, and other reporting output for events, picks, stations, etc. An e-mail or other alert message can be generated for each event with magnitudes or tsunamigenic potential that exceed preset thresholds. Figure A1 shows the main graphical display of Early-est, which summarizes the evolving trace processing, associate/locate module and event characterization results in real time.

\section{Data set}

The Early-est catalog (EEc in this paper) includes fully automatic and unrevised location and magnitude estimations for 5449 events from around the globe recorded at regional and teleseismic distance with magnitude $M \gtrsim 5.0$. The current major version release of Early-est has been running since the beginning of March 2012. Our analysis will use locations and magnitudes for events which occurred between the beginning of March 2012 and the end of December 2014. At the beginning of March 2012, Early-est was using about 300 seismic broadband stations. The number of stations has continuously been increasing, and at the end of September 2014 the Early-est software was using a virtual station network of 494 stations (Fig. 1).

We use the following as reference catalogs: (i) the catalog provided by GEOFON project of the Deutches GeoForschungsZentrum ( $\mathrm{Gc}$ in this paper, http://geofon. gfz-potsdam.de/eqinfo/form.php); (ii) the catalog provided by the US National Earthquake Information Center ( $\mathrm{Nc}$ in this paper); (iii) the catalog provided by the EMSC-CSEM (Cc in this paper) (http://www.emsc-csem.org/Earthquake), (Godey et al., 2007); (iv) the catalog provided by the Global CMT project (CMTc in this work) (Dziewonski et al., 1981; Ekström et al., 2012); (v) and the catalog provided by the Pacific Tsunami Warning Center ( $\mathrm{Pc}$ in this paper) provided to the authors of this paper courtesy of Barry Hirshorn of the Pacific Tsunami Warning Center (http://ptwc.weather.gov). The CMTc and the Pc will be used specifically to compare and assess the $M_{\mathrm{wp}}$ and $M_{\mathrm{wpd}}$ magnitudes.

The above-mentioned observatories and centers provide manually verified and/or revised earthquakes source parameters for different time periods. Table 2 summarizes the abbreviations and time windows for each catalog used in this work. The ICG/NEAMTWS guidelines indicate that tsunami warning must be disseminated for all events in the Mediterranean and northeastern Atlantic regions with $M \geq 5.5$. For this reason, although Early-est locates events with magnitude $M \gtrsim 5.0$, our analysis will focus only on worldwide earthquakes with magnitude $M \geq 5.5$.

\section{Epicenter location}

In this section, we use the three reference catalogs $\mathrm{Nc}, \mathrm{Gc}$, and $\mathrm{Cc}$, and the Early-est catalog EEc.

We first build three couples with the three reference cata$\operatorname{logs}(\mathrm{Gc}-\mathrm{Cc}, \mathrm{Cc}-\mathrm{Nc}$ and $\mathrm{Gc}-\mathrm{Nc})$ and we compute the distance between the epicenter coordinates for each earthquake listed in both catalogs for each couple.

The top panel in Fig. 2 shows the histograms representing the distributions of the location differences in each couple from the reference catalogs. The $M \geq 5.5$ earthquakes are generally located with mean distance differences smaller than $\overline{\delta \Delta_{\text {ref }}} \leqslant 20 \mp 25 \mathrm{~km}$; almost $95 \%$ of all earthquakes are 


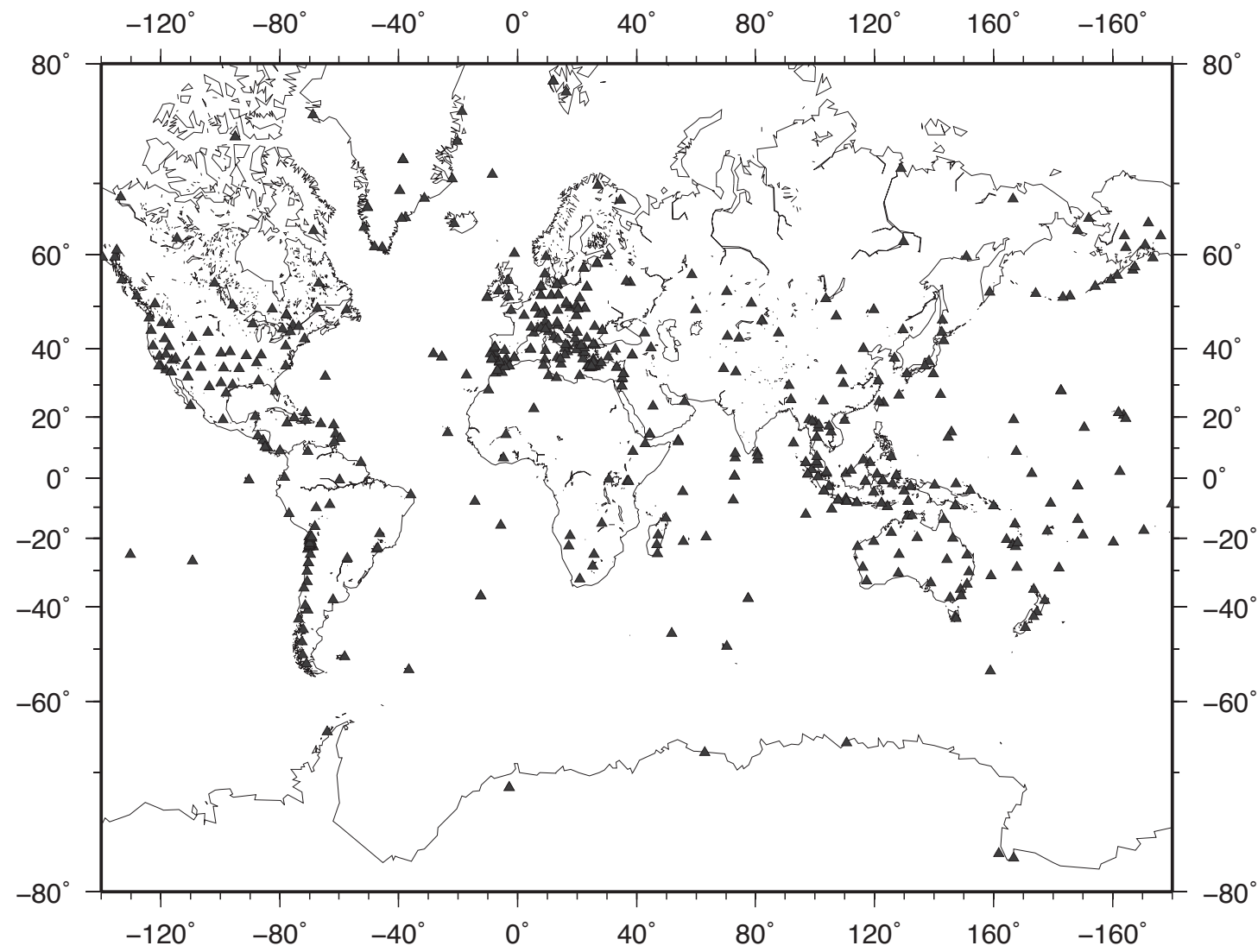

Figure 1. Global map with the 494 seismic broadband stations used by Early-est (the list is updated at the end of September 2014). The stations belong to 45 different networks providing data in real time. When working in real time, latencies in the data stream and/or connection problems may occur, reducing the number of waveform available for location and magnitude estimation.

located with distance differences of $\delta \Delta_{\text {ref }} \lesssim 50 \mathrm{~km}$. We did not find evidence for geographical and/or tectonic dependence of this uncertainty.

We then compare the epicenter coordinates between the earthquakes listed in the EEc and each of the three reference catalogs (Fig. 2, bottom panels), i.e., we build the couples EEc-Cc, EEc-Nc, and EEc-Gc. The histograms show that the epicenter location differences between the EEc and the reference catalogs $\delta \Delta_{\mathrm{EEc}}$ are similar to the differences plotted on the top panels. The mean location difference between the $\mathrm{EEc}$ and the reference catalogs is about $\overline{\delta \Delta_{\mathrm{EEc}}} \lesssim 20 \mp 20 \mathrm{~km}$ and $95 \%$ of all events in the data set show differences $\delta \Delta_{\mathrm{EEc}} \lesssim 45 \mathrm{~km}$.

Generally our analysis showed that earthquakes with $M \geq$ 5.5 can be located by using seismic data from global networks, with an empirical uncertainty, defined as the mean location difference with respect to the reference catalogs, of about $v \approx 20 \mp 25 \mathrm{~km}$.

\section{Hypocenter depth}

In this section we proceed as described in the section above: we use the three reference catalogs $\mathrm{Nc}, \mathrm{Gc}$, and $\mathrm{Cc}$ and the Early-est catalog EEc to build the catalog couples used in the previous section. We then compute the depth difference between the hypocenters for each earthquake listed in both catalogs of each couple.

Figure 3 (top panels) shows histograms that represent the distribution of the depth differences in each couple from the reference catalogs. The hypocenter depth estimation for earthquakes with magnitude $M \geq 5.5$ listed in global cata$\operatorname{logs}$ is generally well resolved: the mean and standard deviations difference are $\overline{\delta Z_{\text {ref }}} \approx 0 \mp 25 \mathrm{~km}$ for all catalog couples. We did not find evidence for geographical and/or tectonic dependence of these differences.

We then compare the hypocenter depths between the EEc and each of the three reference catalogs (Fig. 3 bottom panels; couples EEc-Cc, EEc-Nc, and EEc-Gc). The bottom panels show that the hypocenter depth estimation between the Early-est catalog and the reference catalogs do not differ significantly: the mean difference distributions are about $\overline{\delta Z_{\mathrm{EEc}}} \approx 0 \mp 30 \mathrm{~km}$. 

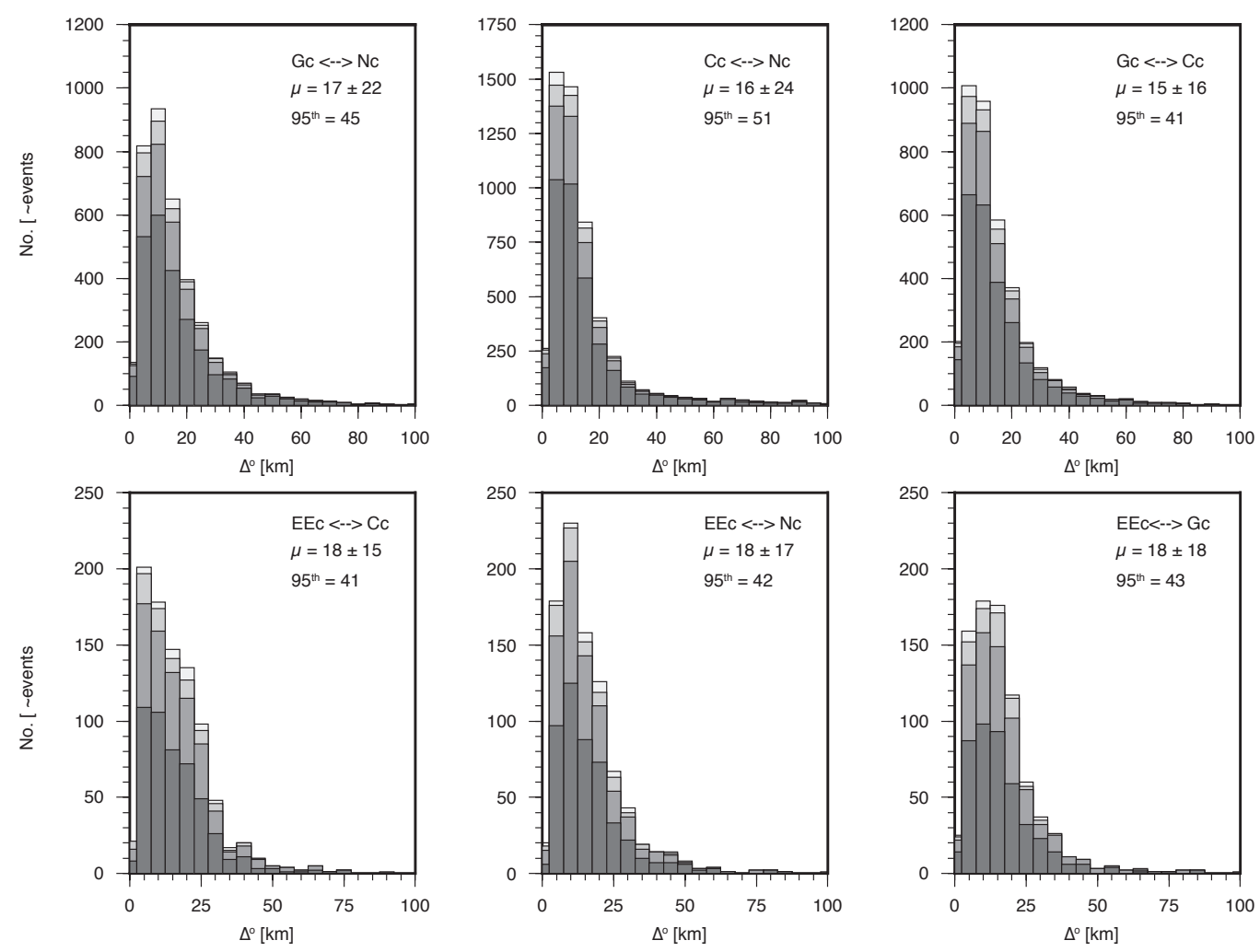

Figure 2. Epicenter location difference distributions for the events listed in the reference and in the Early-est catalogs. The epicenter location difference is expressed in kilometers on the $x$ axis. The $y$ axis refers to the number of events for each bin; the bins are $5 \mathrm{~km}$ each. The top panels show the location difference between the locations of the three reference catalogs: Nc, Gc, and Cc. The bottom panels show the location difference between Early-est and the reference catalogs. The gray color scale indicates magnitude ranges as follows: dark gray $5.5 \leq M<6$, middle dark gray $6.0 \leq M<6.5$, middle light gray $6.5 \leq M<7.0$ and light gray $M \geq 7.0$. The mean and the standard deviation and the $95 \%$ percentiles for the entire dates (i.e., regardless of the magnitude) are indicated on the top right of each panel.

Generally, our analysis showed that hypocenter depth of earthquakes with $M \geq 5.5$ can be precisely estimated when using seismic data from global networks, with an empirical uncertainty of about $v \approx 00 \mp 30 \mathrm{~km}$.

\section{Magnitude}

Early-est provides three different types of magnitude: $\mathrm{mb}$, $M_{\mathrm{wp}}$, and $M_{\mathrm{wpd}}$ (Lomax and Michelini, 2011) and then automatically decides each minute which magnitude type is the most significant, following the rules in Table 3 . The criteria to assign the best magnitude listed in Table 3 follow two simple principles: (i) a minimum number of observations is required to obtain reliable magnitude estimations, and (ii) magnitude types are reliable within magnitude ranges. Following Lomax and Michelini (2009a, b, 2011) we set the validity range $5.8 \leq M_{\mathrm{wp}}<7.2$ for the best magnitude; $\mathrm{mb}$ is assigned to best magnitude when $M_{\mathrm{wp}}<5.8$ and $M_{\mathrm{wpd}}$ is assigned to best magnitude when $M_{\mathrm{wp}}>7.2$ In this work we compare the Early-est magnitude types $M_{\mathrm{wp}}$ and $M_{\mathrm{wpd}}$ with the reference magnitude types $M_{\mathrm{wp}}$, and $M_{\mathrm{w}}$. Since the
Table 3. Early-est computes mb, $M_{\mathrm{wp}}$, and $M_{\mathrm{wpd}}$. This table summarize the rules used by Early-est to define the best magnitude (i.e., the most significative magnitude type) between $\mathrm{mb}, M_{\mathrm{wp}}$, and $M_{\text {wpd }}$. The magnitude mb is computed using the $30 \mathrm{~s}$ time window or the apparent source duration $T_{o}$ as a time window when $T_{o}<30 \mathrm{~s}$ and using the IASPEI WWSSN-SP response for convolution. The magnitude $M_{\mathrm{wp}}$ is scaled to the largest of the first two maxima on integrated displacement within the window from $t_{P}$ to $t_{P}+T_{o}$ time or $120 \mathrm{~s} \mathrm{after} t_{P}$, where $t_{P}$ is the $P$-arrival time - whichever window is the shortest. The magnitude $M_{\text {wpd }}$ (duration-amplitude), which can be viewed as an extension of the $M_{\mathrm{wp}}$ moment-magnitude algorithm, is computed following the $M_{\mathrm{wp}}$ procedure and corrections described in Lomax and Michelini (2012).

\begin{tabular}{lcc}
\hline Best magnitude & $\#^{1}$ & Magnitude range $^{2}$ \\
\hline$M_{\mathrm{wpd}}$ & $\geq 6$ & $M_{\mathrm{wp}} \geq 7.2$ \\
$M_{\mathrm{wp}}$ & $\geq 6$ & $5.8 \leq M_{\mathrm{wp}}<7.2$ \\
$\mathrm{mb}$ & $\geq 6$ & $M_{\mathrm{wp}}<5.8$ \\
\hline 1: number of recording stations with good signal-to-noise \\
ratio and reliable amplitude reading. ${ }^{2}$ : magnitude range \\
validity
\end{tabular}



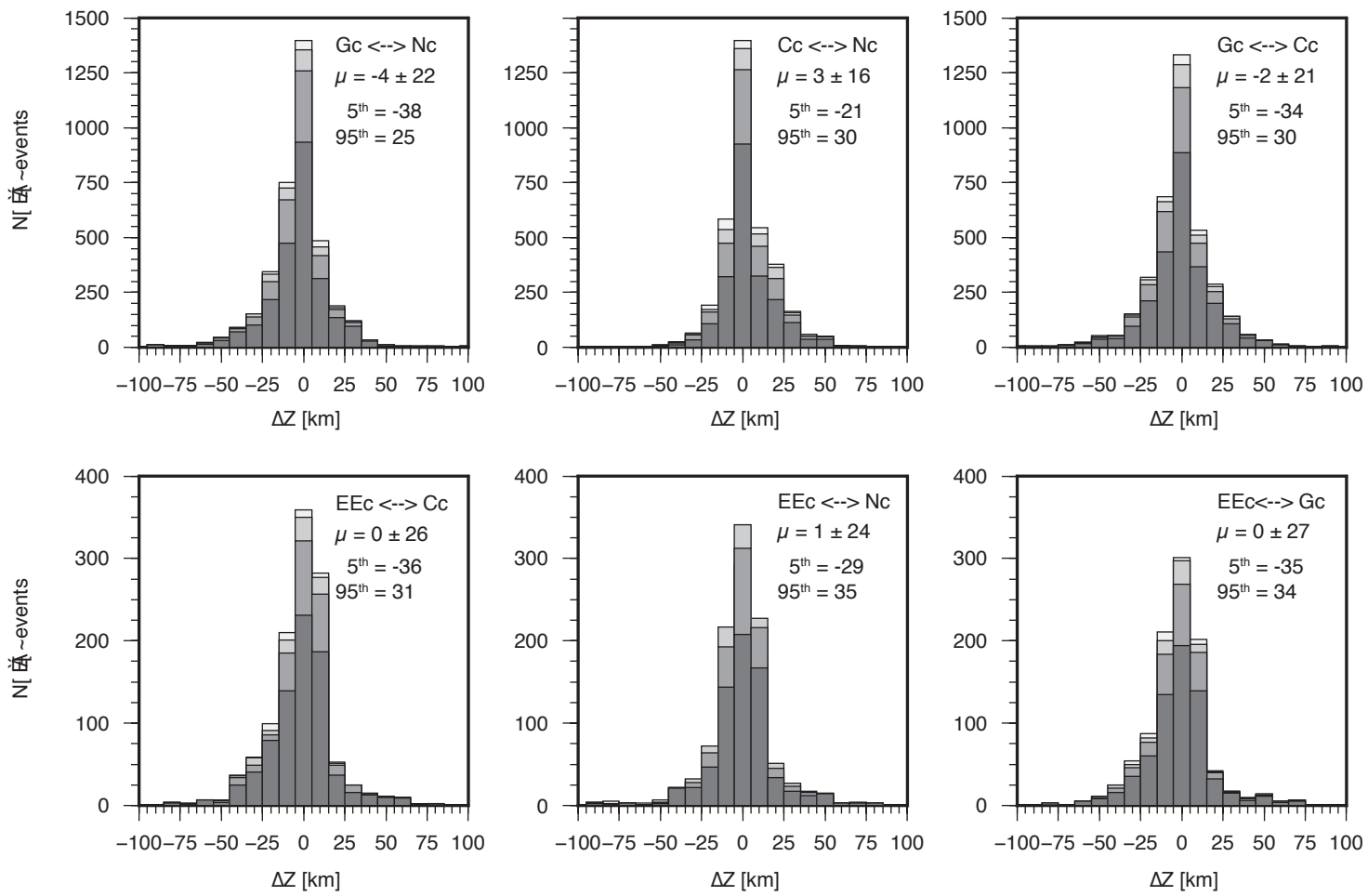

Figure 3. Hypocenter depth difference distributions for the events listed in the reference and in the Early-est catalogs. The hypocenter depth difference is expressed in kilometers on the $x$ axis. The $y$ axis refers to the number of events for each bin; the bins are $5 \mathrm{~km}$ each. The top panels show the hypocenter depth difference distribution between the locations of the three reference catalogs Nc, Gc, and Cc. The bottom panels show the hypocenter depth difference between Early-est and the reference catalogs. The gray color scale indicates magnitude ranges as follows: dark gray $5.5 \leq M<6$, middle dark gray $6.0 \leq M<6.5$, middle light gray $6.5 \leq M<7.0$, and light gray $M \geq 7.0$. The mean and the standard deviation and the $95 \%$ percentiles for the entire dates (i.e., regardless of the magnitude) are indicated on the top right of each panel.

ICG/NEAMTWS guidelines prescribe that for earthquakes with depth $Z \leq 100 \mathrm{~km}$, a standard general warning should only be delivered for events with $M \geq 5.5$, and no action should be taken for smaller magnitudes, we only analyze the magnitude comparisons for events with $Z \leq 100 \mathrm{~km}$ in this section.

As in Sects. 4 and 5 we first compare the magnitudes provided by the reference catalogs. Then, we compare the magnitudes provided by Early-est with the magnitudes listed in the reference catalogs. First we will compare all best magnitude (i.e., $\mathrm{mb}$ or $M_{\mathrm{wp}}$ ) results, only considering couples between catalogs where the magnitude types are identical (Fig. 4). This comparison will provide a general overview on how the best magnitudes of Early-est match with the magnitudes from the reference catalogs.

Figure 4 shows the distribution of the magnitude differences $\delta M^{\mathrm{EEc}}=M^{\mathrm{EEc}}-M^{\text {ref }}$ between the values of the EEc and the ones from the reference catalogs.

When comparing the Early-est magnitudes with the magnitudes from the two reference catalogs (Fig. 4), Early-est seems to overestimate the magnitudes by about $\overline{\delta M^{\mathrm{EEc}}} \approx$ $0.1 \mp 0.2$. The percentiles show that more than $10 \%$ of the magnitudes provided by Early-est differ significantly from the magnitudes provided by the reference catalogs. The overestimation and the wider distribution appear to be homogeneously distributed among all magnitude ranges.

In the next subsections, we will analyze the magnitude values for each single magnitude type mb and $M_{\mathrm{wp}}$ separately in more detail.

\section{$6.1 \mathrm{mb}$}

In this subsection we compare the $\mathrm{mb}^{\mathrm{EE}}$ magnitudes provided by Early-est to the mb magnitudes provided by NEIC $\left(\mathrm{mb}^{\mathrm{Nc}}\right)$ and EMSC $\left(\mathrm{mb}^{\mathrm{Cc}}\right)$. We use the $\mathrm{mb}^{\mathrm{EEc}}$ only when Early-Est assigns the best magnitude to $\mathrm{mb}$, following the rules of Table 3.

Figure 5 shows the $\mathrm{mb}^{\mathrm{EEc}}$ with respect to the $\mathrm{mb}^{\mathrm{Nc}}$ (top left panel) and with respect to the $\mathrm{mb}^{\mathrm{Cc}}$ (top right panel). These two plots show scattered and sparse distributed values, which are coherent with the magnitude differences of the histograms in Fig. $4 \mathrm{c}$ and d. The mean $\overline{\delta \mathrm{mb}}$ indicates that the catalogs are coherent, but the standard deviation and the percentiles point out that the $\mathrm{mb}^{\mathrm{EEc}}$ can be significantly underestimated or overestimated with respect to $\mathrm{mb}^{\mathrm{Nc}}$ and $\mathrm{mb}^{\mathrm{Cc}}$. 

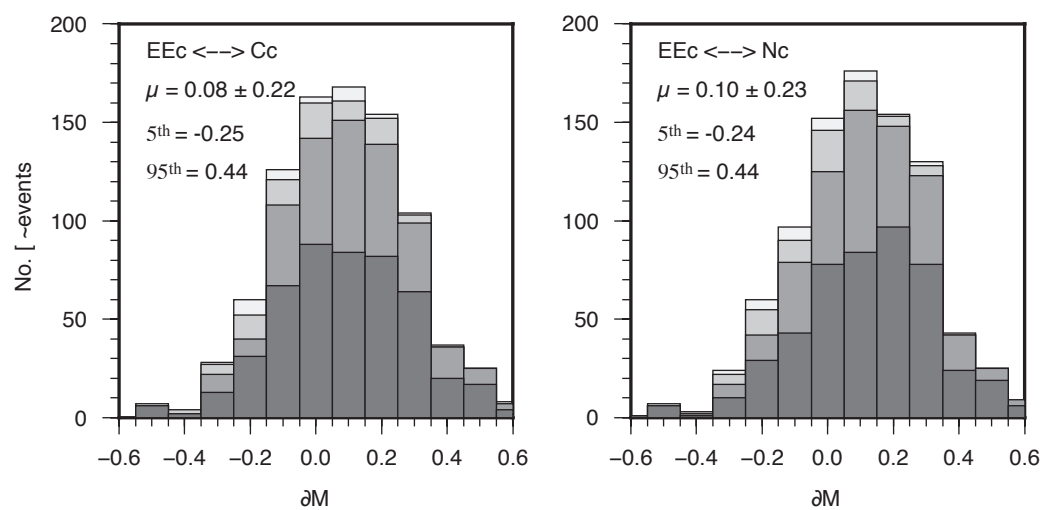

Figure 4. Magnitude difference distributions for the events listed in the EEc catalog compared to the two Nc and Cc reference catalogs. Differences are computed only when the same magnitude type is provided for the same event in the two compared catalogs. The magnitude difference is on the $x$ axis. The $y$ axis refers to the number of events for each bin; the bins are 0.1 magnitude each. The color scale refers to the same magnitude ranges as in Figs. 3 and 2, and not to the magnitude type. The gray color scale indicate magnitude ranges as follows: dark gray $5.5 \leq M<6$, middle dark gray $6.0 \leq M<6.5$, middle light gray $6.5 \leq M<7.0$, and light gray $M \geq 7.0$. The mean and the standard deviation and the $95 \%$ percentiles for the entire dates (i.e., regardless of the magnitude) are indicated on the top left of each panel.

In order to correct such scattered and sparse distribution, we computed a linear regression function for each panel (thick dashed lines on the top panels). These functions are computed for $f_{1}=\mathrm{mb}^{\mathrm{EEc}} \rightarrow \mathrm{mb}^{\mathrm{Nc}}$ and for $f_{2}=\mathrm{mb}^{\mathrm{EEc}} \rightarrow$ $\mathrm{mb}^{\mathrm{Cc}}$. The constant $a$ and $b$ of the linear function are shown in the upper left corners of Fig. 4a and b. We then applied the regression functions $f_{1}$ and $f_{2}$ to the $\mathrm{mb}^{\mathrm{EEc}}$ values and we recompute the differences (third row of the histograms). Both new distributions have mean values close to 0 and smaller standard deviation and percentiles compared to the original ones.

The two functions appear similar but show different $a$ and $b$ constants. In order to test if such differences are significant, first we applied the function $f_{1}$, derived for $\mathrm{mb}^{\mathrm{EEc}} \rightarrow \mathrm{mb}^{\mathrm{Nc}}$, and we computed the differences with respect to the $\mathrm{mb}^{\mathrm{Cc}}$ values. Secondly, we applied the function $f_{2}$, derived for $\mathrm{mb}^{\mathrm{EE}} \rightarrow \mathrm{mb}^{\mathrm{Cc}}$, and we computed the residuals with respect to the $\mathrm{mb}^{\mathrm{Nc}}$ values. Applying these corrections, we obtain two new difference distributions: $\delta \mathrm{mb}_{\mathrm{Cc}}^{\mathrm{EEc} \rightarrow \mathrm{Nc}}$ and $\delta \mathrm{mb}_{\mathrm{Nc}}^{\mathrm{EE} \rightarrow \mathrm{Cc}}$ (bottom left and right panels). The $\delta \mathrm{mb}^{\mathrm{EE} \rightarrow \mathrm{Nc}}$ and $\delta \mathrm{mb}_{\mathrm{Cc}}^{\mathrm{EEc} \rightarrow \mathrm{Nc}}$ distributions, and the $\delta \mathrm{mb}^{\mathrm{EE} \rightarrow \mathrm{Cc}}$ and the $\delta \mathrm{mb}_{\mathrm{Nc}}^{\mathrm{EE} \rightarrow \mathrm{Cc}}$ distributions appear to be significantly different. We performed a $t$ test between $\delta \mathrm{mb}^{\mathrm{EE} \rightarrow \mathrm{Nc}}$ and the $\delta \mathrm{mb}_{\mathrm{Cc}}^{\mathrm{EEc} \rightarrow \mathrm{Nc}}$ distribution and between $\delta \mathrm{mb}_{\mathrm{Cc}}^{\mathrm{EEc} \rightarrow \mathrm{Nc}}$ and $\delta \mathrm{mb}_{\mathrm{Nc}}^{\mathrm{EEc} \rightarrow \mathrm{Cc}}$. The null hypothesis $H_{o}$ is rejected at more than $95 \%$.

From the percentiles of the corrected distributions, particularly on the left side, we observe that when applied, the regression function $f_{1}$ produces a narrower magnitude difference distribution with respect to the function $f_{2}$.

Generally, after applying the linear corrections, the resulting $\mathrm{mb}^{\mathrm{EE}}$ uncertainty $(v \approx 0.00 \mp 0.14)$, with respect to the reference catalogs, is coherent with the overall magnitude uncertainty between the two reference catalogs (Fig. 4, left panel).

\section{$6.2 \quad M_{\mathrm{wp}}$}

As a reference, we first compare the magnitudes $M_{\mathrm{wp}}^{\mathrm{Pc}}$ values provided by the Pacific Tsunami Warning Center (PTWC) using the correction of Whitmore et al. (2002) with the $M_{\mathrm{w}}^{\mathrm{CMTc}}$ of the CMT-Harvard catalog (Fig. 6). The magnitudes compare well with a mean difference $\mu=0.04 \mp 0.19$ for events with magnitude about $M_{\mathrm{wp}} \lesssim 7.0-7.5$. For larger events, the magnitudes $M_{\mathrm{wp}}^{\mathrm{Pc}}$ begin to overestimate with respect to the $M_{\mathrm{w}}^{\mathrm{CMTc}}$.

We now compare the magnitudes $M_{\mathrm{wp}}^{\mathrm{EEc}}$ with the $M_{\mathrm{w}}^{\mathrm{CMTc}}$ (Fig. 7). The $M_{\mathrm{wp}}^{\mathrm{EEc}}$ magnitudes appear to be significantly overestimated ( $>0.2$ magnitude unit) for earthquakes with $M_{\mathrm{w}}^{\mathrm{CMT}} \leq 6.5$.

$M_{\mathrm{wp}}$ is based on the far-field approximation to the $P$ wave displacement due to a double-couple point source (Tsuboi et al., 1995), thus we should consider that the result of $M_{\mathrm{wp}}$ computed in the field near to the source may be biased. In fact Hirshorn et al. (2012) showed that single station $M_{\mathrm{wp}}$ values measured at stations at epicentral distances $\Delta \leq 15^{\circ}$ have positive residuals with respect to the Harvard centroid moment tensor $M_{\mathrm{w}}$. Nevertheless, our procedure is built to obtain reliable $M_{\mathrm{wp}}$ estimates as fast as possible, thus we aim to also use $M_{\mathrm{wp}}$ measured from stations close to the epicenter.

To test if our $M_{\mathrm{wp}}^{\mathrm{EEc}}$ values may be dependent as a function of the distance between station and epicenter, we plotted the station residuals at each station for each event with respect to the epicenter distance (Fig. 8). Station residuals are defined as $\delta M_{\mathrm{wp}}^{i}=M_{\mathrm{wp}}^{\mathrm{EEc}, i}-M_{\mathrm{w}}^{\mathrm{CMTc}}$, where $i$ indicates the $M_{\mathrm{wp}}$ values measured at each station. 
(a)
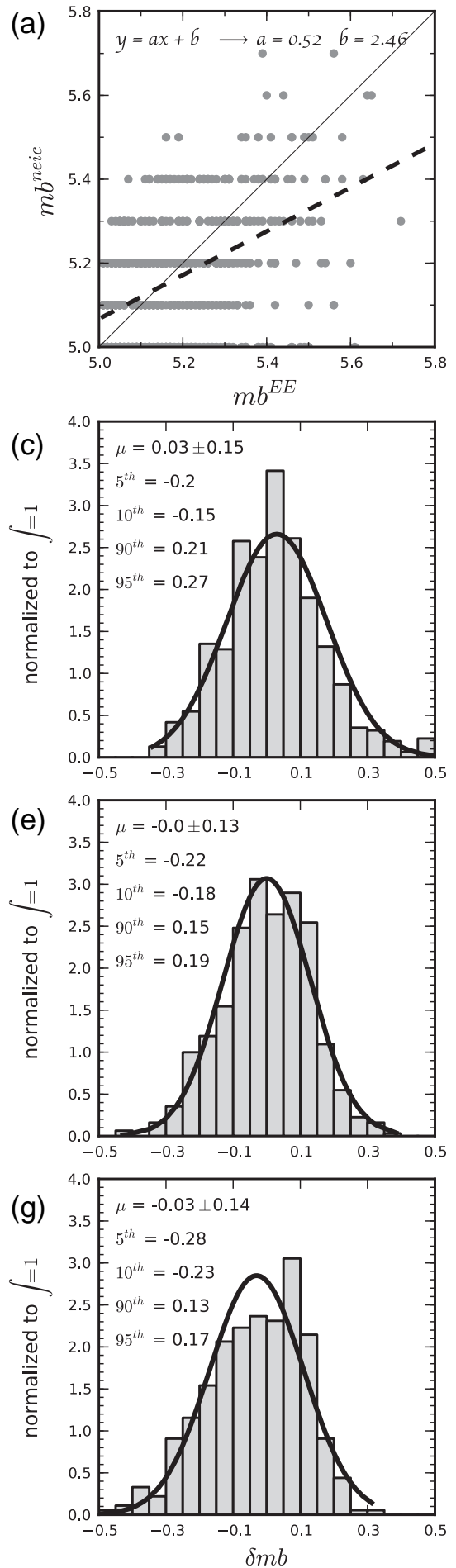

(b)

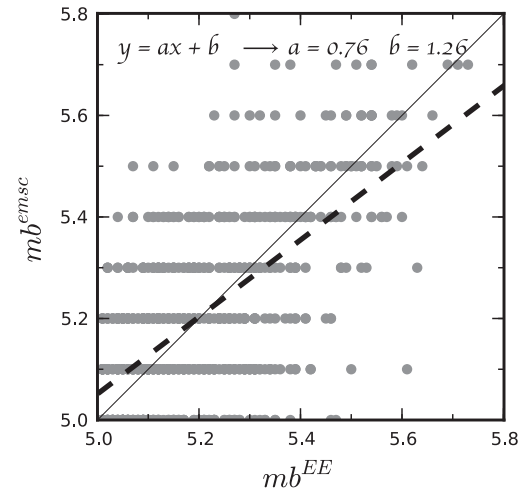

(d)

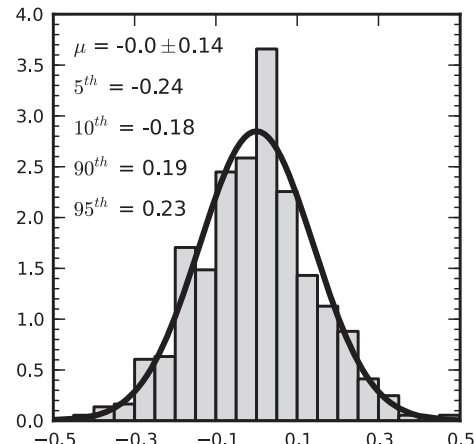

(f)

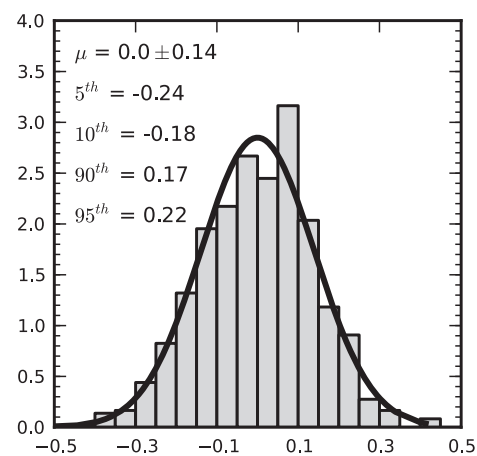

(h)

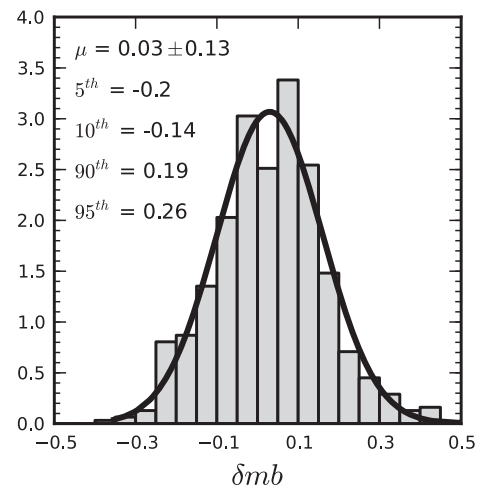

Figure 5. Magnitude mb differences between the Early-est catalog and the reference catalogs (Nc on the left and Cc on the right). Top row panels (a) and (b) depict $m b$ magnitude comparison between the Early-est values ( $x$ axis) and the reference catalog values ( $y$ axis). The dashed lines refer to the linear regression functions, the $a$ and $b$ constant are indicated on the upper left corner, and the thin black line refers to the $1: 1$ proportion. Second row panels (c) and (d) depict mb magnitude difference distribution; each bin is 0.05 magnitude units wide. The black line refers to the theoretical distribution derived from measured mean $\mu$ and standard deviation $\sigma$ with $\int=1$. Third row panels (e) and (f) are as in the second row panels, but after applying the correction function, shown in the top panels, to the Early-est mb. Fourth row panels (g) and (h) are as in the third row panels, but on the left panel, the EEc-Cc derived correction is applied and on the right panel, the EEc-Nc derived correction is applied. 

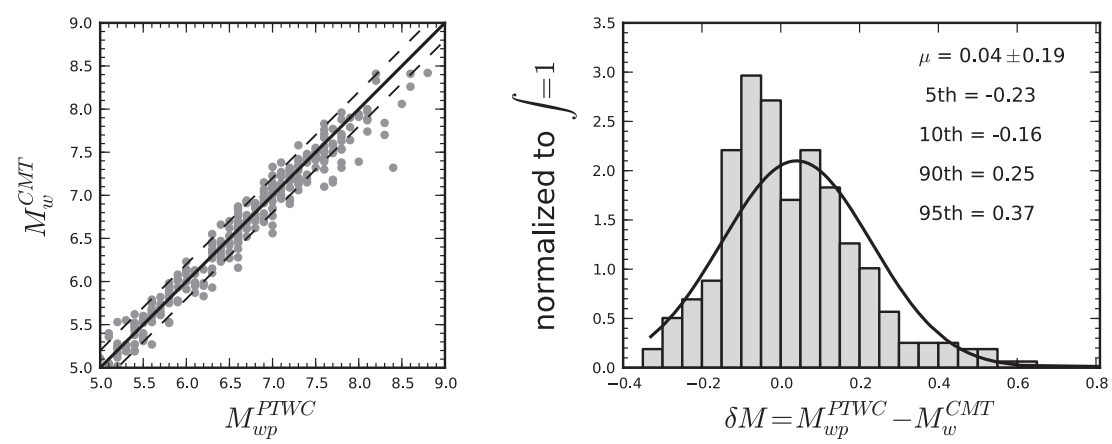

Figure 6. Comparison between the $M_{\mathrm{wp}}$ magnitudes computed by the Pacific Tsunami Warning Center (PTWC) and the $M_{\mathrm{W}}$ magnitudes from the CMT-Harvard catalog. Plot on the left side: the dots denote magnitude values, the continuous line denotes the $1: 1$ ratio, and dashed lines denote $\mp 0.2$ uncertainty. The histogram on the right side shows the $\delta M_{\mathrm{wp}}-M_{\mathrm{w}}$ distribution. Mean, standard deviation, and percentiles are indicated on the top right of the right panel. Each bin is 0.05 magnitude wide.

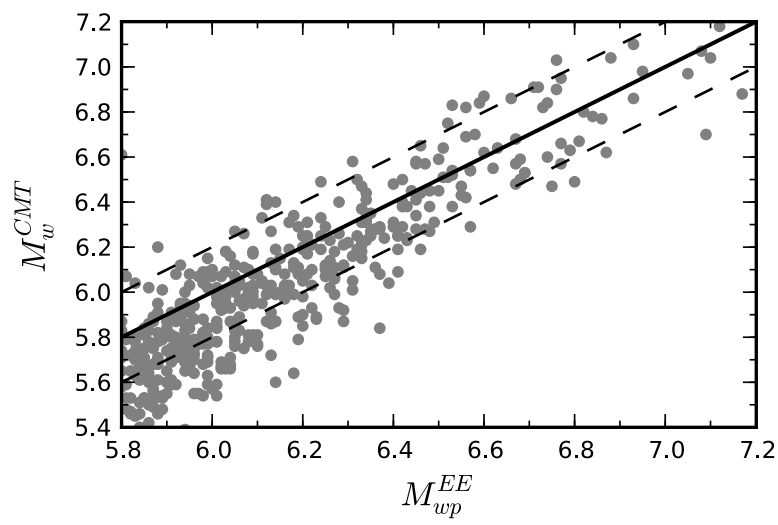

Figure 7. Early-est magnitudes $M_{\mathrm{wp}}$ compared with the CMTHarvard $M_{\mathrm{W}}$ from the CMT-Harvard catalog. The continuous line indicates the $1: 1$ ratio and dashed lines indicate $\mp 0.2$ uncertainty.

The top left of Figure 8 shows the residuals $\delta M_{\mathrm{wp}}^{i}$ (gray dots) for all events with hypocenter depth $\leq 100 \mathrm{~km}$ plotted with respect to the epicentral distance in degrees. From these residuals we compute the regression function (dashed line in Fig. 8):

$$
\begin{aligned}
f(\Delta)= & -1.32 e^{-6} \cdot \Delta^{3}+2.40 e^{-4} \cdot \Delta^{2} \\
& -0.0146 \cdot \Delta+0.314
\end{aligned}
$$

Figure 8 and Eq. (1) show that the $\delta M_{\mathrm{wp}}^{i}$ are overestimated for distances $\Delta \lesssim 30^{\circ}$ and slightly underestimated for distances $\Delta \gtrsim 90^{\circ}$. After applying the regression function $f(\Delta)$ to the station values, the distance dependency of $M_{\mathrm{wp}}^{i}$ is removed (Fig. 8 top right panel).

The distance dependency of the measured $M_{\mathrm{wp}}^{\mathrm{EEc}, i}$ at each station results in a general overestimation of the $M_{\mathrm{wp}}^{\mathrm{EEc}}$ with respect to the $M_{\mathrm{w}}^{\mathrm{CMTc}}$ (Fig. 7 bottom left). The overestimation of $M_{\mathrm{wp}}^{\mathrm{EEc}}$ could of course be removed using only $M_{\text {wp }}$, measured at stations with epicentral distance $30^{\circ} \leq$ $\Delta \leq 90^{\circ}$. Nevertheless, Early-est is designed to provide au- tomatic magnitude estimations within a few minutes after the event origin time in order to disseminate early tsunami warnings. Thus the closer stations are relevant and must be used.

For this reason we apply Eq. (1) to remove the distance dependency of the measured $M_{\mathrm{wp}}^{\mathrm{EEc}, i}$ and we then recompute the magnitude events $M_{\mathrm{wp}, \text { corr }}^{\mathrm{EEc}}$. To recompute the $M_{\mathrm{wp} \text {, corr }}^{\mathrm{EEc}}$ we follow the Early-est procedure as follows: we cut off stations with $M_{\mathrm{wp}}^{\mathrm{EEc}, i}<10$ th percentile and with $M_{\mathrm{wp}}^{\mathrm{EEc}, i}>10$ th percentile. The event magnitude is $M_{\mathrm{wp}}=50$ th percentile of the remaining values. The bottom right histogram of Fig. 8 shows the corrected magnitude differences $\delta M_{\mathrm{wp} \text {, corr }}^{\mathrm{EEc}}$ The right shift of the original magnitude differences distribution (Fig. 8, bottom left) is corrected. The resulting magnitude $M_{\mathrm{wp}}^{\mathrm{EEc}}$ uncertainty with respect to the $M_{\mathrm{w}}^{\mathrm{CMTc}}$ is $\delta M_{\mathrm{wp}}=$ $0.0 \mp 0.2$, which is consistent with the uncertainty of the $M_{\text {wp }}$ provided by the PTWC with respect to the global CMTHarvard catalog.

\section{Speed performance and tsunami warning alert timeline}

In the previous section, we analyzed the final epicenter location, hypocenter depth, and magnitude values provided by Early-est, i.e., the values obtained about $20 \mathrm{~min}$ to $1 \mathrm{~h}$ after the event origin time. A tsunami alert however, is meaningful when delivered within a short time after the event origin time and with reliable earthquake source parameters. In order to plan the timeline procedure at the CAT-INGV, we want to know how fast the earthquake source parameters computed by Early-est converge toward a stable value.

We thus first analyze how fast Early-est provides a first automatic location, and second, how fast the epicenter coordinates and the magnitudes stabilize.

The histogram in Fig. 9 shows the delay time after the event origin time when a first automatic location of Early-est becomes available. We generally have to wait at least $2 \mathrm{~min}$ in order to have a first automatic solution; within 7 and $10 \mathrm{~min}$ 

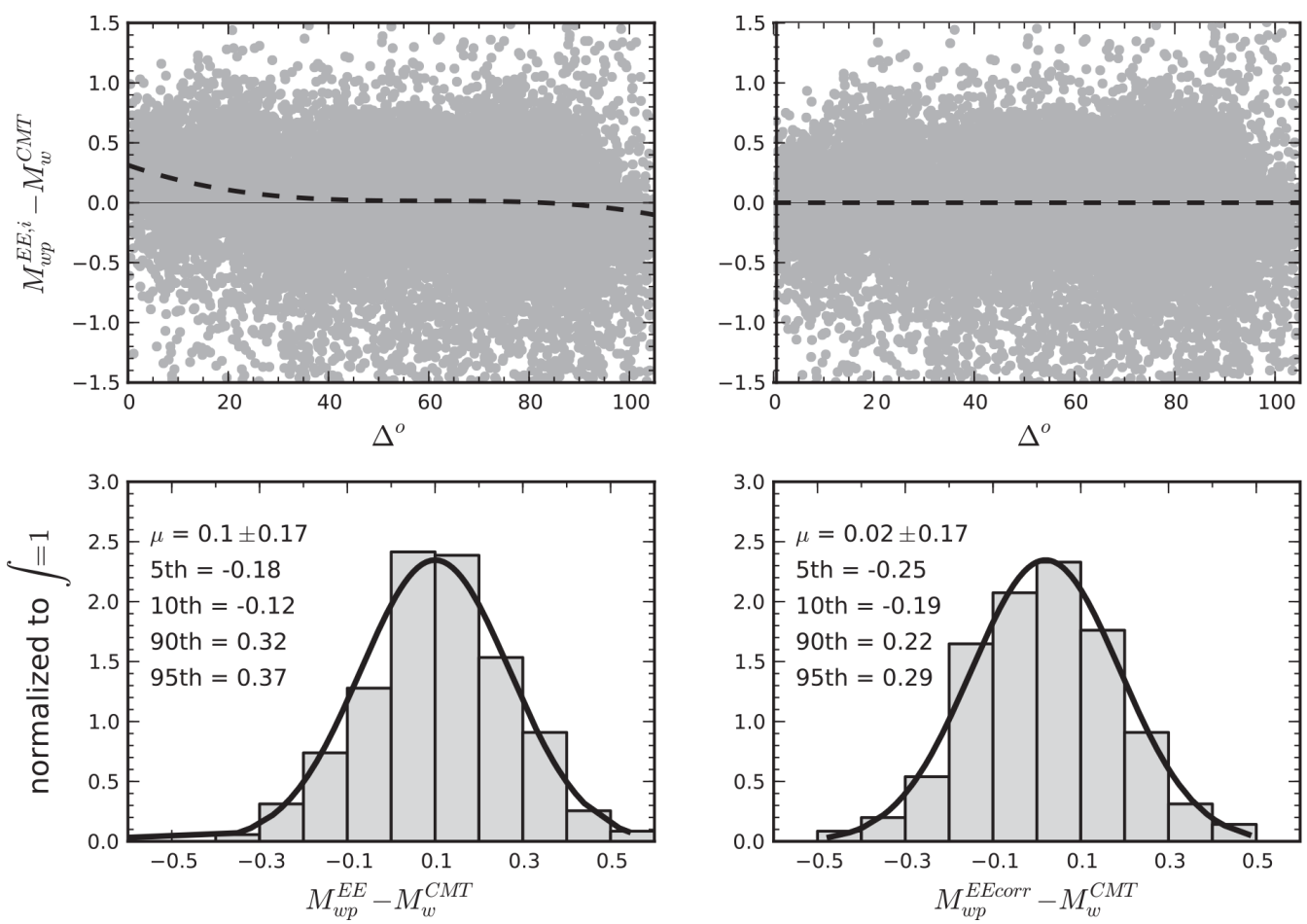

Figure 8. Epicentral distance dependence of the $M_{\mathrm{wp}}$ for events with hypocentral depth $\leq 100 \mathrm{~km}$. The top left panel shows station residuals $\delta M_{\mathrm{wp}}^{i}=M_{\mathrm{wp}}^{\mathrm{EE}, i}-M_{\mathrm{w}}^{\mathrm{CMT}}$ (gray dots), plotted with respect to the epicentral distance in degree, and the dashed line, which represents a third degree polynomial regression function (Eq. 1) which best fits the data. The top right panel indicates station residuals $\delta M_{\mathrm{wp}}^{i}=M_{\mathrm{wp}}^{\mathrm{EEcorr}, i}-$ $M_{\mathrm{W}}^{\mathrm{CMT}}$ (gray dots), after applying the regression function (Eq. 1), plotted with respect to the epicentral distance in degree, and the dashed line, which is a third degree polynomial regression function, which best fits the corrected residuals with respect to the distance. Bottom left panel: event magnitude difference $\Delta M_{\mathrm{wp}}$ distribution before the distance correction. These distribution are similar to Fig. 7 as follows: mean, standard deviation, and percentiles are indicated on the left of the histogram, bins are 0.5 magnitude wide each, and the black solid line refers to theoretical distribution with $\int=1$. The bottom right panel shows event magnitude difference $\Delta M_{\mathrm{wp}}^{\text {corr }}$ distribution after the distance correction using Eq. (1).

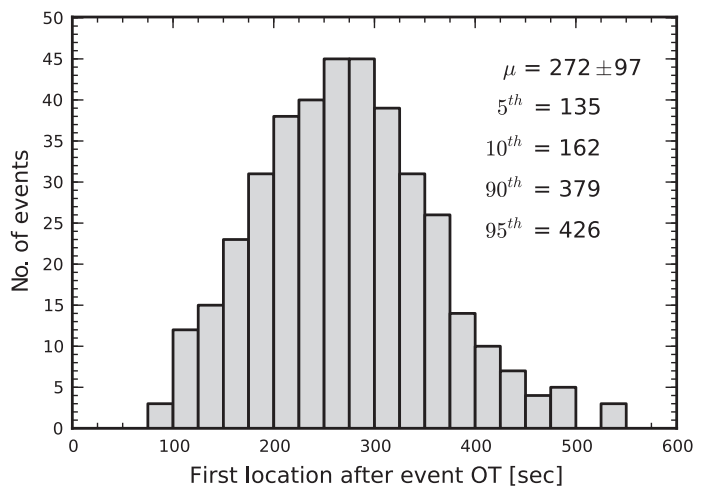

Figure 9. Early-est first location performance. This figure shows how fast a first location for global events is available through Earlyest. The bins ( $25 \mathrm{~s}$ wide) on the $x$ axis refer to the seconds after the event origin time (OT) when a first location is available. On the top right, the mean, the standard deviation, and four representative percentiles are indicated. after the event origin time, about 95 and $100 \%$ of all earthquakes are located, respectively. On a global scale, a large number of earthquakes are located along the oceanic ridges and trenches, which are far away from most of the seismic stations. In the Mediterranean region, the distances between earthquake sources and seismic stations are generally shorter than on a global scale. Table 1 lists the 12 events with magnitude $M \geq 5.5$ that occurred in the Mediterranean region between March 2012 and the end of December 2014. These 12 events do not form a reliable statistic, but from Table 1 we may reasonably expect to locate an event in the Mediterranean region with magnitude $M \geq 5.5$ within 2-3 min after the event origin time.

Figure 10 shows how fast a first location (top panel) and magnitude (bottom panel) stabilizes towards the final and stable values.

Both panels indicate that for most of the events, the epicenter coordinates and magnitudes within the first 8-10 min after the first available location may be considered stable and significantly close to the final values, since the magnitudes are 

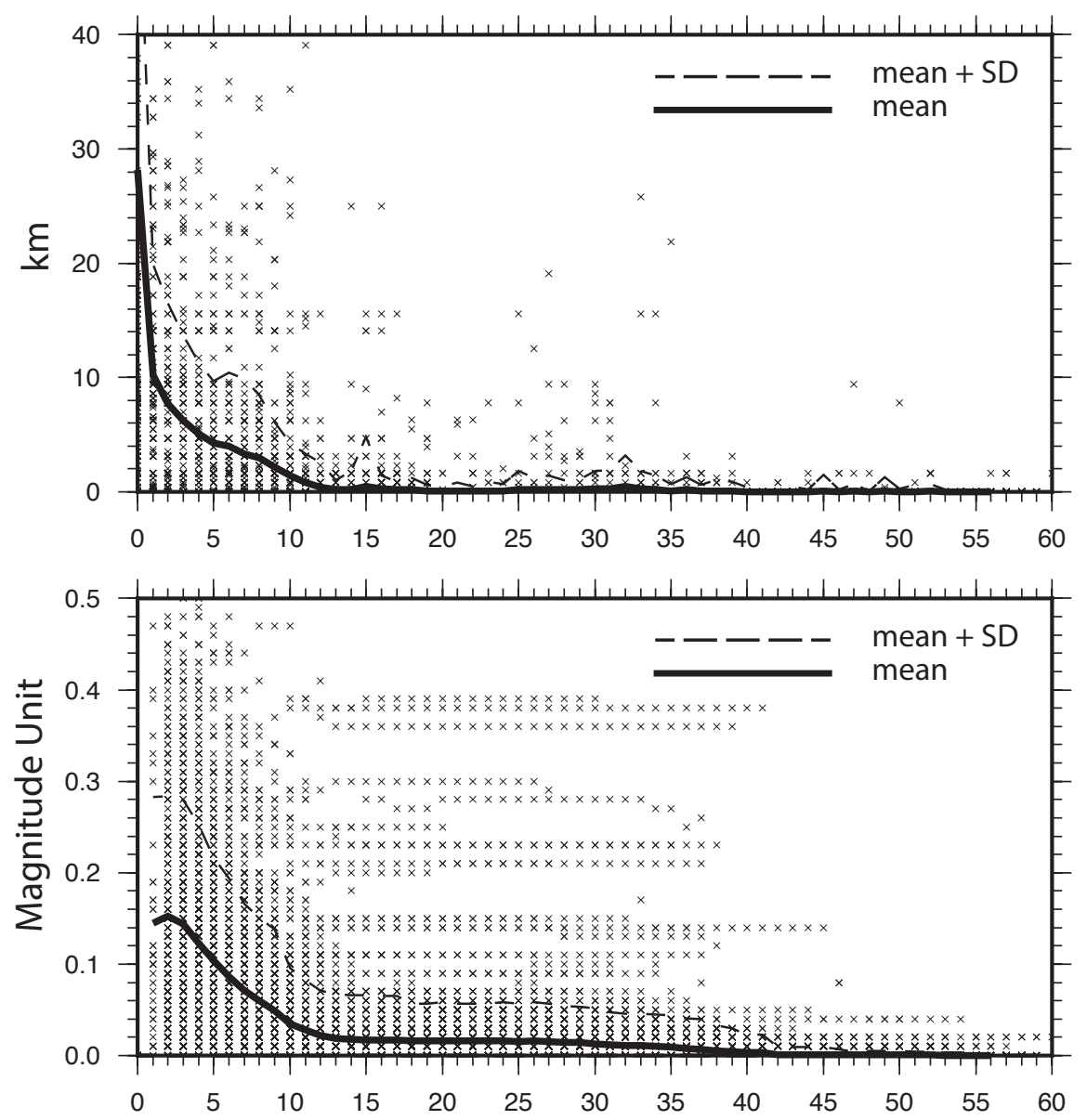

Figure 10. Early-est location and magnitude estimation stability performances. This figure shows how fast a first location estimation (top panel) and magnitude (bottom panel) estimations evolve towards stable values. Top panel: for each run, we computed the distance in kilometers between the current epicenter and the epicenter of the last location. Bottom panel: for each run we computed the absolute magnitude difference between the current magnitude and the final magnitude. In this panel, most of the magnitudes are available 2 min after the event origin time, since often the first automatic location may not provide a magnitude value. The magnitude refers to the "best" magnitude decided by Early-est (Table 3) at each run. In both panels difference values (depicted by black crosses) are plotted on the $y$ axis with respect to the minutes after the first location is established ( 0 value at the $x$ axis). The black line depicts the mean value computed for each minute and the dashed line shows the mean plus the standard deviation.

$\mu+\sigma \leq 0.2$ and the epicenter locations are $\mu+\sigma \leq 10 \mathrm{~km}$, respectively.

The CAT-INGV uses the earthquake source parameters provided by Early-est to compile the tsunami warning messages to be disseminated to the civil authorities. The mission of the CAT is to provide tsunami warnings for earthquakes with $M \geq 5.5$ which occur in the Mediterranean region according to the ICG/NEAMTWS guidelines.

Based on the speed performances of Early-Est on computing reliable earthquake source parameters (Fig. 10) and on the minimum delay time after the event origin time to localize an event in the Mediterranean (Table 1), we set the timeline described below to allow fast enough production of warning messages based on robust seismic estimates.

Based on Fig. 10 we decided to automatically compile a tsunami warning alert message always for the second, the fifth and the eighth locations available after the first location is established. Considering that the first location in the Mediterranean region may be available within 2-3 min after the event origin time, the second, the fifth and the eighth locations may be available by about 5,8 , and $11 \mathrm{~min}$ after the event origin time. Therefore, in the case of an earthquake in the Mediterranean region, the continuous monitoring of Early-est provides information to the seismologists for issuing tsunami warnings. Based on Fig. 10 and Table 1, such a procedure may be executed within about $\approx 15 \mathrm{~min}$ after the event origin time. The messages are delivered via several media such as mail, fax, GTS (https://www.wmo.int/pages/prog/ www/TEM/GTS), and SMS. This ensures that messages are typically delivered to authorities within seconds. 


\section{Discussions and final remarks}

Early-est is able to provide a first location within about 7 min from the origin time for almost $95 \%$ of all worldwide earthquakes. In the Mediterranean region, where the epicentral distance between the earthquake and the seismic station is smaller, we may expect a first automatic location result within 2-3 min after the event origin time. Generally within less than $10 \mathrm{~min}$ after the first location is established, the estimations converge to stable values.

In our analysis, the automatic locations and source depth estimates provided by Early-est for global $M \geq 5.5$ earthquakes are robust and reliable; in fact the epicenter source parameters estimates by Early-est are coherent with the epicenter source parameters provided after manual revision/validation by other agencies (NEIC, GFZ, and CSEMEMSC) that locate earthquakes on a global scale.

Generally our analysis showed that earthquakes with $M \geq$ 5.5 can be located by using seismic data from global networks, with an empirical uncertainty, defined as the mean location difference with respect to the reference catalogs, of about $v \approx 20 \mp 25 \mathrm{~km}$. The locations provided by Early-est show differences to the locations from the reference catalogs, that are comparable to the location differences among the reference catalogs.

A similar conclusion is valid for the mean Early-est focal depth difference for global $M \geq 5.5$ earthquakes, which is about $v \approx 0 \mp 25 \mathrm{~km}$, which is also coherent with the focal depth differences between the reference catalogs.

Early-est uses only a subset of all worldwide, public, realtime stations, and the fact that the available number of stations sometimes may be reduced because of latencies does not seem to affect the quality of the estimated epicenter coordinates and hypocenter depth.

The magnitude is a key earthquake parameter to determine the tsunami alert level (see Sect. 1). The decision matrix defined by the NEAMTWS (2011) sets the tsunami warning level on the basis of the magnitude, hypocenter depth, and the distance between the epicenter and the coastal forecast points. The automatic mb and $M_{\mathrm{wp}}$ magnitudes provided by Early-est show differences to the reference values used, that in some cases may be significant in the context of a tsunami warning.

The mb magnitudes provided by Early-est compare well with the mb values provided by reference catalogs from the point of view of the mean differences, but show sparse and scattered distributions that can be larger than $\mp 0.3$ units of magnitude. Such sparse distribution can be corrected by increasing the signal-to-noise ratio threshold for the mb station values. On the other hand, a higher signal-to-noise ratio threshold may reduce the number of station readings, and would require more stations to obtain a reliable mb value. This would result in a slower magnitude estimation, which may affect the efficiency and the speed required for the dissemination of tsunami warnings. A linear correction of the computed mb values produces indeed a reduction of the standard deviation to about $\mp 0.15$ units of magnitude. Both $f_{1}$ and $f_{2}$ corrections help the avoidance of large magnitude over- and underestimations. The $f_{1}$ correction function shows slightly more narrow distribution than the $f_{2}$ correction function.

Nevertheless, the mb magnitude starts to saturate from a magnitude of $\mathrm{mb} \gtrsim 6.0$, and for this reason Early-est does not use mb when $M_{\mathrm{wp}} \geq 5.8$. Thus, mb values apply to earthquakes which are not generally expected to be tsunamigenic.

The Early-est magnitude $M_{\mathrm{wp}}$ values are reliable when computed using only stations with epicentral distance $30^{\circ} \leq \Delta \leq 90^{\circ}$. As expected (Tsuboi et al., 1995; Hirshorn et al., 2012), single station $M_{\mathrm{wp}}^{i}$ measurements at distance $\Delta \leq 30^{\circ}$ are significantly overestimated (Fig. 8). The observed distance-dependent bias at each station results in a general overestimation of the final $M_{\mathrm{wp}}$ (Fig. 7). Earlyest is designed to provide automatic magnitude estimation within a few minutes after the event origin time in order to disseminate early tsunami warnings, thus the closer stations are relevant and must be used. For this reason we prefer to correct the station $M_{\mathrm{wp}}$ values to remove the overestimation of the single station $M_{\mathrm{wp}}$ values at distance $\Delta \leq 30^{\circ}$, instead of introducing a minimum distance cut off.

Since the assignment rules for the best magnitude depend on the number of stations measuring reliable $\mathrm{mb}, M_{\mathrm{wp}}$, and $M_{\text {wpd }}$ and the magnitude value for each one (Table 3), the assigned best magnitude may vary between $\mathrm{mb}, M_{\mathrm{wp}}$, and $M_{\text {wpd }}$ at each run. This is particularly true within the first few minutes after the event origin time, when the number of available waveforms may still be small, and the magnitude values may not be stable yet (Fig. 10). The linear correction for $\mathrm{mb}$ and the distance-dependent correction for $M_{\mathrm{wp}}$ will thus produce a stable and reliable best magnitude useful for seismologically based tsunami early warning procedures.

The CAT-INGV provides seismologically based tsunami early warnings when earthquakes with magnitude $M \geq 5.5$ occur in the Mediterranean region. Such tsunami warning messages are based on the fully automatically location and magnitude estimations provided by the Early-est software. The analysis of a data set of 3 years of worldwide earthquakes showed that Early-est is a robust, reliable, and efficient piece of software for automatic real-time earthquake source parameter estimation, which provides reliable and robust location parameters and magnitude estimations within a few minutes after the event origin time. 


\section{Appendix A: Octree associate/locate module}

The octree associate/locate module (Fig. A2) efficiently and robustly associates picks, and detects and locates seismic events over the whole Earth from 0 to $700 \mathrm{~km}$ depth using the efficient, nonlinearized, probabilistic, and global octree importance-sampling search (Lomax et al., 2001, 2009). The objective function for the octree search is a probability function, $P(\boldsymbol{x})$, based on the stacking of implicit origin times for each pick for each potential source $\boldsymbol{x}_{\text {test }}$. Given a seismic wave velocity model (currently ak135 Kennett et al., 1995), a pick time $t_{\mathrm{p}}$ at a seismic station, and assuming a seismic phase type that may have produced the pick, the phase traveltime from the source $\boldsymbol{x}_{\text {test }}$ to the station $T_{x}$ can be calculated and thus the implicit origin time $T_{0}$ for the source and phase can be determined by back projection (e.g., $T_{0}=T_{\mathrm{p}}-T_{x}$ ). The set of stacks of $T_{0}$ for all picks forms a histogram over potential origin times for a source at $\boldsymbol{x}_{\text {test }}$. If the maximum histogram value exceeds a specified threshold, and if the associated picks for the maximum pass tests on amplitudes and station distributions, then $P\left(x_{\text {test }}\right)$ is retained to drive the octree search further to find a maximum $\boldsymbol{x}_{\max }=\max [P(\boldsymbol{x})]$ and define a seismic event at $\boldsymbol{x}_{\max }$ and associated picks.

The octree search is direct and nonlinearized - it does not involve linearization of the equations relating the pick times to the source location, and is global and probabilistic; it samples throughout the prior probability density function (PDF) for the seismic location problem. The search uses an initial, coarse, regular grid-search followed by recursive, octal sub-division, and sampling of cells in three-dimensional, latitude/longitude/depth space to generate a cascaded, octree structure of sampled cells. The octree search produces approximate importance-sampling - the spatial density of sampled cells follows the objective function $P$.

For each latitude/longitude/depth cell of volume $v$ scanned by the octree search, a histogram-like stack over implicit origin times for first-arrival, $P$ phases (currently Pg, $P$, Pdiff, PKPdf), is constructed for all picks in the pick list. Each origin-time value $T_{0}$ is assigned a distance and pick-qualityweighted amplitude A between 0 and 1.0, and an uncertainty $\sigma$ determined by the sum of half the maximum travel-time range across the cell volume with the travel-time and pick uncertainties. Each implicit origin time is included in the origin-time stack with amplitude A using two step-function time limits at $T_{0} \mp \sigma$ inserted in time order. After all picks have been processed, the maximum of the origin-time stack is found by a systematic scan over the available time limits; the use of step-function time limits and time ordering makes this scan very fast. All picks whose origin time limits overlap the stack maximum time are flagged as associated. The stack value, combined with the variance of the implicit origin times from all associate picks, is converted to a probability, $P(\boldsymbol{x}, v)$. If the maximum stack value exceeds a specified threshold (currently 4.5), and if the associated picks for the maximum pass tests on amplitude attenuation, station dis- tance, and azimuth distributions, then $P(\boldsymbol{x}, v)$ is stored for use in the progression of the octree search. If any of these conditions are not met, then the octree associate/locate module returns with a flag that no event has been associated. $P(x, v)$ represents the relative probability that an event is located within a cell of volume $v$ at position $\boldsymbol{x}$.

The octree search to associate/locate is paused when the subdivided cells reach an adaptively determined, minimum size (e.g., $\leq 5 \mathrm{~km}$ for a location constrained by regional to globally distributed stations, $\leq 1 \mathrm{~km}$ for a location constrained by locally distributed stations); in this pause, uncertainty measures (e.g., PDF scatter samples) are generated in the association stage. The octree search and cell subdivision is then continued for a fixed number of samples (currently about 4600 ) to obtain a refined, precise location by fixing the associated phases to those corresponding to the maximum of the $P(\boldsymbol{x}, v)$ found in the association stage. The fixing of the associated phases is necessary for small cell sizes since a decreasing cell volume combined with the step-function limits on origin time leads to a continuous reduction in $P(x, v)$ values and eventual instability and nonconvergence of the octree search near and at the optimal source location. The precise octree results provide uncertainty measures (e.g., PDF scatter samples, uncertainty ellipsoid) for the location.

When the octree associate/locate module returns an event, the associated picks for this event are masked in the pick list and the octree associate/locate module is run again using the remaining, non-associated picks, until no further events are returned. Thus multiple events can be associated and located within a report interval, and, in general, the events are identified in order of the number of associated picks and better location constraint.

Early-est runs the octree associate/locate module every 1 min using all picks from the past hour, without knowledge of or preserving information from previously associations and event locations. This procedure makes Early-est relatively simple algorithmically, and robust with regards to changes in the set of available picks and the number of associated picks defining locations. In particular, this procedure allows early stage locations with few associated picks to easily move in space or origin time, or to split in multiple events, or to be absorbed in other events, or to disappear as more pick data become available. However, this procedure is inefficient for later stage event locations which are defined by a larger number of associated picks, e.g., more than 1020 picks, since such locations are very unlikely to change; much processing effort is repeated each minute to reobtain a previous result. This inefficiency can be problematic after large earthquakes, when the repeated re-processing of hundreds of picks from a mainshock and large aftershock can cause Early-est to fall behind real time. 


\section{Early-est Earthquake Monitor - Graphical Display: Overview}

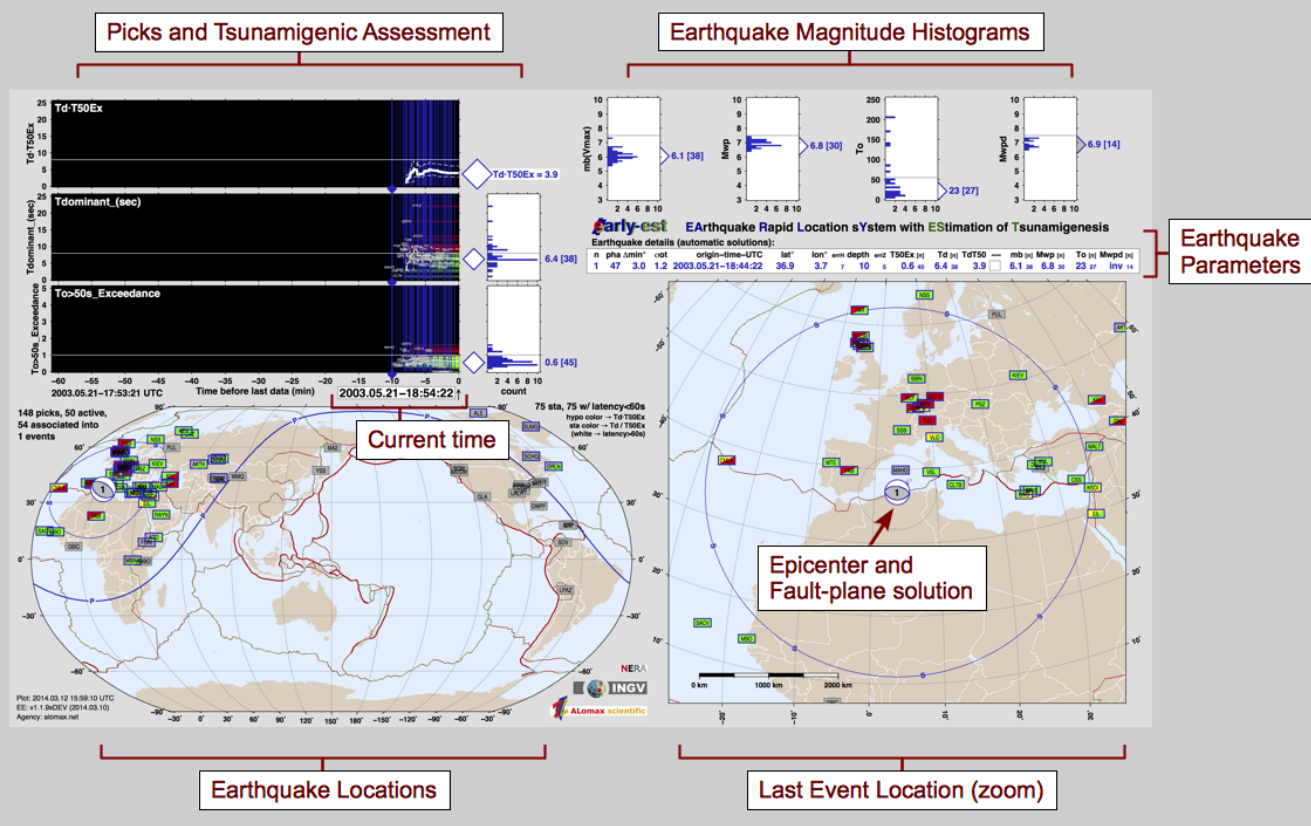

\section{Early-est Earthquake Monitor - Graphical Display: Event Location}

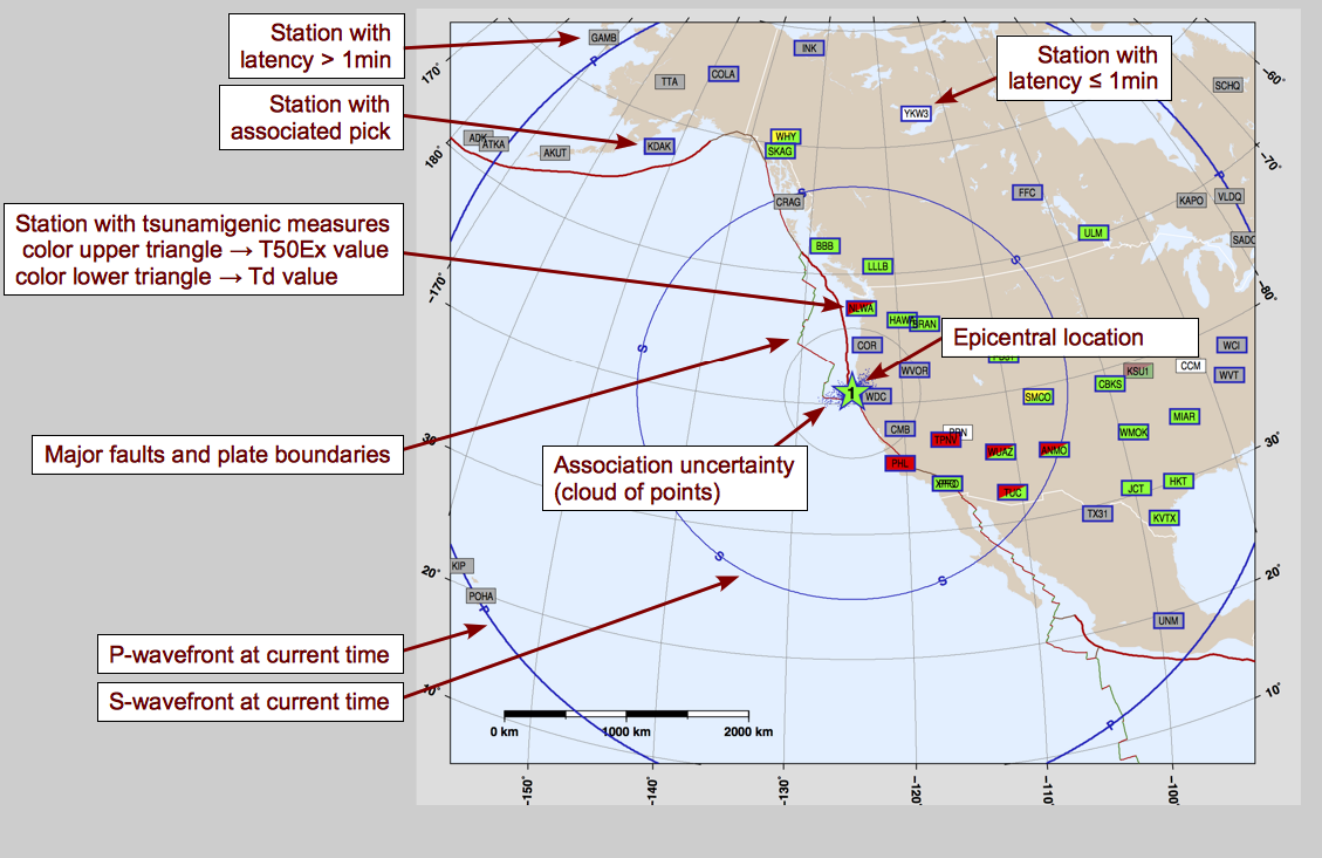

Figure A1. Main graphical display of Early-est. 


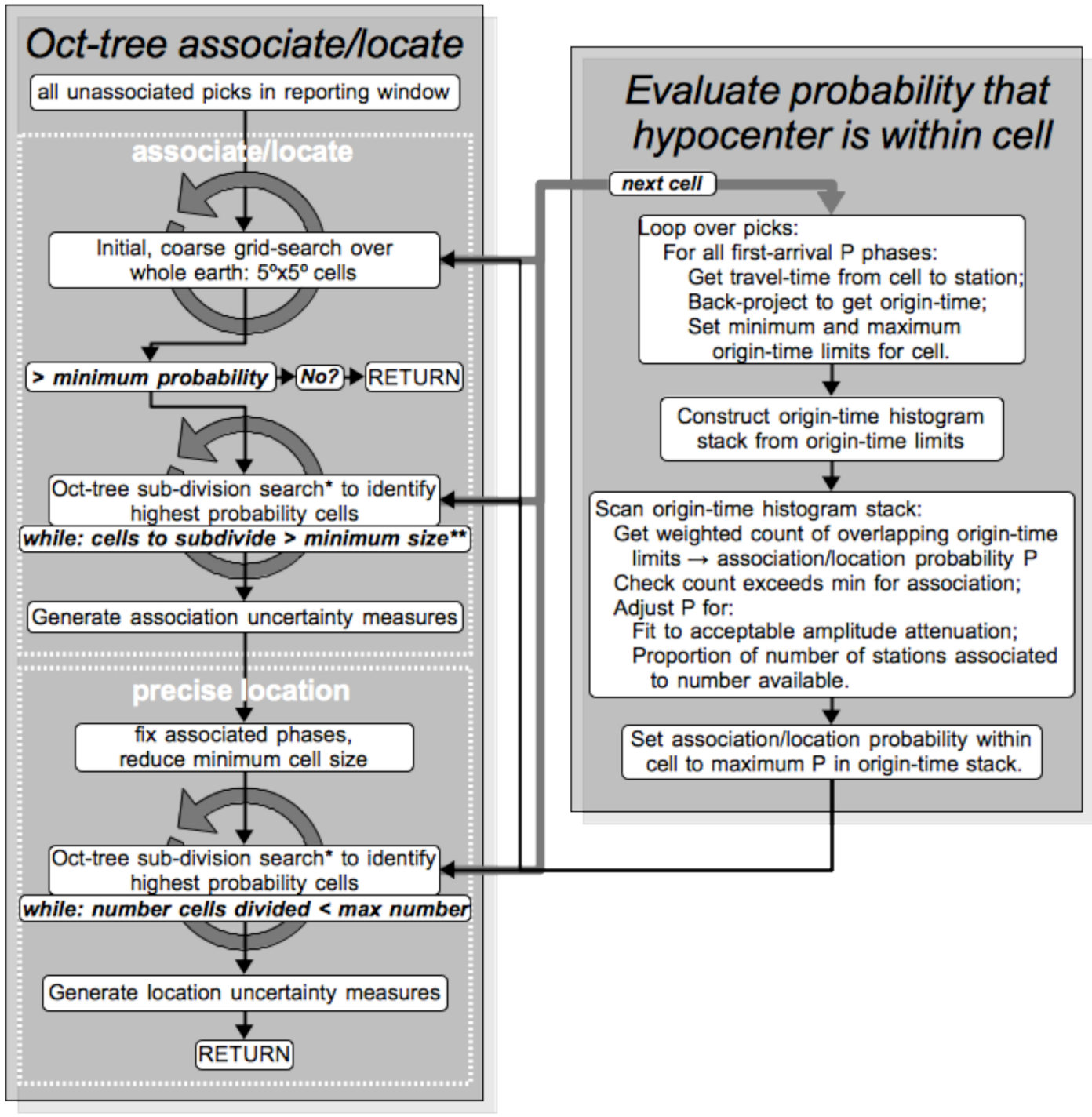

Figure A2. Early-est associate/locate flow diagram: * Cell division is performed at a fixed cell size for a specified number of cells or until no cell available to divide; the fixed cell size is then reduced and cell division continued. ${ }^{* *}$ minimum size is adaptively reduced in proportion to number of associated stations near epicenter. 
Table A1. Early-est parameter specifications.

\begin{tabular}{|c|c|c|}
\hline Measure & References & Description, modifications \\
\hline $\mathrm{Td}$ & Lomax and Michelini (2011) & Max. dominant period smoothed over $5 \mathrm{~s}$ in window from $T_{\mathrm{p}}$ to $T_{\mathrm{p}}+55$. \\
\hline$T_{50} \mathrm{Ex}$ & Lomax and Michelini (2011) & $\begin{array}{l}T_{50} \text { exceedance, modified as follows: } \\
\quad \text { - reduced } T_{50} \text { Ex minimum distance to } 5^{\circ}\end{array}$ \\
\hline $\mathrm{Td} \cdot T_{50} \mathrm{Ex}$ & Lomax and Michelini (2011) & $\begin{array}{l}\text { Period-duration discriminant for tsunami potential, modified as follows: } \\
\text { - reduced } \mathrm{Td} \cdot T_{50} \mathrm{Ex} \text { minimum distance to } 5^{\circ}\end{array}$ \\
\hline$T_{o}$ & Lomax and Michelini (2009a, b, 2011) & $\begin{array}{l}\text { High frequency, apparent source duration, modified as follows: } \\
\text { - removed smoothing window width of } 10 \mathrm{~s} \text { from } T_{o} \text { for short durations, applied with a } \\
\text { linear ramp from } 10 \rightarrow 0 \mathrm{~s} \text { for initial durations of } 20 \rightarrow 60 \mathrm{~s} \text { and minimum duration is at } \\
\text { its highest frequency in } \mathrm{HF} \text { stream }(0.2 \mathrm{~s}) \\
\text { - reduced } T_{o} \text { minimum distance to } 5^{\circ} \\
\text { - added reference of } T_{o} \text { duration to } S \text { arrival time }\left(T_{S}\right) \text { if raw duration end time } T_{o, \text { end }} \text { is } \\
\left.\text { after } T_{S} \text { (e.g., if } T_{o, \text { end }}>T_{S}+\left(T_{S}-T_{\mathrm{p}}\right) / 3 \text { then } T_{o}=T_{o, \text { end }}-T_{S}\right) \text {. }\end{array}$ \\
\hline
\end{tabular}

$\mathrm{mb}\left(V_{\max }\right) \quad$ Bormann and Saul $(2008,2009)$

mb body wave magnitude using $V_{\max }$ formulation:

- apply a recursive, time-domain filter to BRB velocity that implements the WWSSN-SP displacement response

- WWSSN-SP displacement response from Working Group on Magnitudes (Magnitude WG) of the International Association of Seismology and Physics of the Earth's Interior (IASPEI) Commission on Seismological Observation and Interpretation (CoSOI) 2011

This filter is applied to the BRB velocity, so effectively gives: integrate $\rightarrow$ simulate the WWSSN-SP response $\rightarrow$ differentiate, without doing the integration and differentiation.

- measure $V_{\mathrm{max}}$ - the peak from $T_{\mathrm{p}}$ to the lesser of $T_{\mathrm{p}}+T_{o}$ or $T_{\mathrm{p}}+30 \mathrm{~s}$

- apply: $\operatorname{mb}\left(V_{\max }\right)=\log _{10}\left(V_{\max } / 2 \pi\right)+Q(\Delta, h)$

\begin{tabular}{|c|c|c|}
\hline$M_{\mathrm{wp}}$ & Tsuboi et al. $(1995,1999)$ & $\begin{array}{l}M_{\mathrm{wp}} \text { magnitude, modified as follows: } \\
\quad-\text { applied from } T_{p} \text { to the lesser of } T_{\mathrm{p}}+T_{o} \text { or } T_{\mathrm{p}}+120 \mathrm{~s}\end{array}$ \\
\hline$M_{\mathrm{wpd}}(R T)$ & Lomax and Michelini (2009a) & $\begin{array}{l}M_{\mathrm{wpd}} \text { duration-amplitude, large earthquake magnitude, modified as follows to allow simple } \\
\text { and robust real-time application without event type determination: } \\
\text { - use constant } k=4.213 e 19 ; \text { PREM depth correction; no geometrical spreading or attenua- } \\
\text { tion corrections. } \\
\text { - moment correction applied to all event types if } T_{o}>80 \mathrm{~s} \\
\text { - moment correction applied to all event types if } T_{o}>80 \mathrm{~s} \\
\text { - reduced } M_{\text {wpd }} \text { minimum distance to } 5^{\circ} \text { (stable since added reference of } T_{o} \text { duration to } T_{S} \text { ) }\end{array}$ \\
\hline $\begin{array}{l}\text { Focal } \\
\text { mecha- } \\
\text { nism. }\end{array}$ & & $P$ arrival, first-motion focal mechanism using the HASH program. \\
\hline $\begin{array}{l}\text { Focal } \\
\text { mecha- } \\
\text { nism. }\end{array}$ & Hardebeck and Shearer (2002) & $\begin{array}{l}\text { Probabilistic, } P \text { arrival, first-motion and amplitude focal mechanism algorithm (fmamp). Uses } \\
\text { octree search; solution quality based on weighted distribution (quasi-PDF) of } P \text { and } T \text { axis. } \\
\text { (note: under development; not included yet in Early-est distribution.) }\end{array}$ \\
\hline
\end{tabular}


Acknowledgements. We thank the two referees P. Roudil and F. Haslinger for their reviews and advice, and the Editor A. Armigliato for his careful proof-reading. The magnitude $M_{\mathrm{wp}}$ parameters of the Pc used in this paper were provided to the authors courtesy of Barry Hirshorn of the Pacific Tsunami Warning Center. This work has been funded by the Italian Flagship Project RITMARE, by the EU FP7 project NERA (262330), and by project ASTARTE (Assessment, Strategy And Risk Reduction for Tsunamis in Europe) FP7-ENV2013 6.4-3, grant 603839. The Early-est software is being further developed in the framework of the agreement between Italian DPC and INGV, annex B2 (2015). Figures are produced using GMT (Wessel and Smith, 1995) and python matplotlib plotting library. We used broadband seismograms provided by the IRIS DMC, the NEIC-USGS, the IPGP Data Center, the GFZ Seismological Data Archive, the USGS and the INGV and MedNet seismic networks.

Edited by: A. Armigliato

Reviewed by: F. Haslinger and P. Roudil

\section{References}

Basili, R., Tiberti, M. M., Kastelic, V., Romano, F., Piatanesi, A., Selva, J., and Lorito, S.: Integrating geologic fault data into tsunami hazard studies, Nat. Hazards Earth Syst. Sci., 13, 10251050, doi:10.5194/nhess-13-1025-2013, 2013.

Bormann, P. and Saul, J.: The new IASPEI standard broadband magnitude $m b$, Seism. Res. Lett., 79, 698-705, doi:10.1785/gssrl.79.5.698, 2008.

Bormann, P. and Saul, J.: Earthquake magnitude, in: Encyclopedia of Complexity and Systems Science, edited by: Meyers, A., Springer, New York, doi:10.1007/978-0-387-30440-3_151, 2009.

Bungum, H. and Lindholm, C.: Tsunamigenic Seismic Sources in the North Sea, the Norwegian Continental Margin and the Norwegian-Greenland Sea, Tech. rep., NORSAR, 2007.

Dziewonski, A. M., Chou, T.-A., and Woodhouse, J. H.: Determination of earthquake source parameters from waveform data for studies of global and regional seismicity, J. Geophys. Res., 86, 2825-2852, 1981.

Ekström, G., Nettles, M., and Dziewonski, A. M.: The global CMT project 2004-2010: Centroid-moment tensors for 13,017 earthquakes, Phys. Earth Planet. Int., 200, 1-9, doi:10.1016/j.pepi.2012.04.002, 2012.

Godey, S., Bossu, R., Guilbert, J., and Mazet-Roux, G.: The EuroMediterranean Bulletin: A comprehensive seismological Bulletin at regional scale, Seism. Res. Lett., 77, 460-474, 2007.

Hardebeck, J. L. and Shearer, P. M.: A new method for determining first-motion focal mechanisms, Bull. Seism. Soc. Am., 92, 22642276, 2002.

Hirshorn, B., Weinstein, S., and Tsuboi, S.: On the Application of $M_{w p}$ in the Near Field and the March 11, 2011 Tohoku earthquake, Pure Appl. Geophys., 170, 975-991, doi:10.1007/s00024012-0495-3, 2012.

Jenny, S., Goes, S., Giardini, D., and Kahle, H.-G.: Earthquake recurrence parameters from seismic and geodetic strain rates in the eastern Mediterranean, Geophys. J. Int., 157, doi:10.1111/j.1365-246X.2004.02261.x, 2004.
Kagan, Y. Y. and Jackson, D. D.: Tohoku earthquake: A Surprise?, Bull. Seism. Soc. Am., 103, 1181-1194, doi:10.1785/0120120110, 2013.

Kennett, B. L. N., Engdahl, E. R., and Buland, R.: Constraints on seismic velocities in the Earth from travel times, Geophys. J. Int., 122, 108-124, 1995.

Koravos, G. C., Main, I. G., Tsapanos, T. M., and Musson, R. M. W.: Maximum earthquake magnitudes in the Aegean area constrained by tectonic moment release rates, Geophys. J. Int., 152, 94-112, 2003.

Lomax, A. and Michelini, A.: $M_{w p d}$ : A Duration-Amplitude Procedure for Rapid Determination of Earthquake Magnitude and Tsunamigenic Potential from P Waveforms, Geophys. J. Int., 176, 200-214, doi:10.1111/j.1365-246X.2008.03974.x, 2009a.

Lomax, A. and Michelini, A.: Tsunami early warning using earthquake rupture duration, Geophys. Res. Lett., 36, L09306, doi:10.1029/2009GL037223, 2009b.

Lomax, A. and Michelini, A.: Tsunami early warning using earthquake rupture duration and P-wave dominant period: the importance of length and depth of faulting, Geophys. J. Int., 185, 283291, doi:10.1111/j.1365-246X.2010.04916.x, 2011.

Lomax, A. and Michelini, A.: Tsunami early warning within 5 minutes, Pure Appl. Geophys., 170, 1385-1395, doi:10.1007/s00024-012-0512-6, 2012.

Lomax, A. and Curtis, A.: Fast, probabilistic earthquake location in 3D models using oct-tree importance sampling, Geophys. Res. Abstr., 3, 955, 2001.

Lomax, A., Michelini, A., and Curtis, A.: Earthquake Location, Direct, Global-SearchMethods, Encyclopedia of Complexity and Systems Science, part. 5, 2449-2473, doi:10.1007/978-0-38730440-3_150, 2009.

Lorito, S., Selva, J., Basili, R., Tiberti, M. M., and Piatanesi, A.: Probabilistic hazard for seismically induced tsunamis: accuracy and feasibility of inundation maps, Geophys. J. Int., 200, 574588, doi:10.1093/gji/ggu408, 2015.

Maramai, A., Brizuela, B., and Graziani, L.: The EuroMediterranean Tsunami Catalogue, Ann. Geophys., 57, S0435, doi:10.4401/ag-6437, 2014.

NEAMTWS: Interim Operational Users Guide for the Tsunami Early Warning and Mitigation System in the North-eastern Atlantic, the Mediterranean and Connected Seas (NEAMTWS), Version 2.0, ICG/NEAMTWS-VIII, 2011.

Tsuboi, S., Takano, K. A., and Yamanaka, Y.: Rapid determination of $M_{w}$ from broadband $\mathrm{P}$ waveforms, Bull. Seism. Soc. Am., 85, 606-613, 1995.

Tsuboi, S., Takano, K. A., and Yamanaka, Y.: Application of $M_{w p}$ to deep and teleseismic earthquakes, Bull. Seism. Soc. Am., 89, 1345-1351, 1999.

Vassallo, M., Satriano, C., and Lomax, A.: Automatic picker developments and optimization: A strategy for improving the performances of automatic phase pickers, Seism. Res. Lett., 83, 541554, doi:10.1785/gssrl.83.3.541, 2012.

Wessel, P. and Smith, W. H. F.: New version of the generic mapping tool released, Trans. Am. Geophys. Union, p. 329, 1995.

Whitmore, P. M., Tsuboi, S., Hirshorn, B., and Sokolowski, T. J.: Magnitude-dependent correction for Mwp, Sci. Tsunami Hazard., 20, 187-192, 2002. 